\title{
A Coloured Petri Net Framework for Modelling Aircraft Fleet Maintenance
}

\author{
Jingyu Sheng ${ }^{1}$, Darren Prescott ${ }^{2}$ \\ 1. 93582 Troops, Chinese Air Force, Shanxi, 038300, China \\ 2. Resilience Engineering Research Group, University of Nottingham, Nottingham, NG7 2RD,UK
}

\begin{abstract}
The aircraft fleet maintenance organisation is responsible for keeping aircraft in a safe, efficient operating condition. Through optimising the use of maintenance resources and the implementation of maintenance activities, fleet maintenance management aims to maximise fleet performance by, for example, ensuring there is minimal deviation from the planned operational schedule, that the number of unexpected failures is minimised or that maintenance cost is kept at a minimum. To obtain overall fleet performance, the performance of individual aircraft must first be known. The calculation of aircraft performance requires an accurate model of the fleet operation and maintenance processes. This paper aims to introduce a framework that can be used to build aircraft fleet maintenance models. A variety of CPN (coloured Petri nets) models are established to represent fleet maintenance activities and maintenance management, as well as the factors that have a significant impact on fleet maintenance including fleet operation, aircraft failure logic and component failure processes. Such CPN models provide an ideal structured framework for Monte Carlo simulation analysis, within which calculations can be performed in order to determine numerous fleet reliability and maintenance performance measures.
\end{abstract}

Keywords: Maintenance, Decision Support Systems, Aircraft Fleet, Modelling Framework, Coloured Petri Nets

\section{Introduction}

Maintenance is often described as a set of actions carried out to restore a system or vehicle to the state in which it is able to continuously perform its intended functions or missions [1]. It relies on a variety of factors such as equipment, spares and personnel. In fleets of vehicles, such as aircraft, maintenance can be seen as a group of interrelated support structures whose purpose is to minimise interruptions to fleet operations. With this in mind, aircraft fleet maintenance is generally performed with the aim of increasing aircraft reliability by decreasing the number of unexpected aircraft failures and reducing the downtime of inoperative aircraft by ensuring effective repairs.

Generally, aircraft maintenance can be split into two main types: corrective maintenance (CM) and preventive maintenance (PM). CM, also sometimes referred to as reactive or breakdown maintenance, includes all the maintenance actions that return unserviceable aircraft or components to operative states following an unscheduled arising. It is carried out after a failure and due to the random nature of failures cannot be planned, meaning that it is also often referred to as unscheduled maintenance. The primary objective of PM is to prevent unscheduled downtime or failures that would result in CM. PM is an effective way to extend the life of aircraft systems. PM can generally be divided into two categories: time-based and condition-based, which differ according to the variable used to decide when maintenance should be performed. Under a time-based PM procedure, maintenance tasks are performed at fixed, pre-determined intervals, which can be defined in terms of calendar time, flight time or cycles of operation. By contrast, condition-based PM, also known as predictive maintenance, takes place when the regular or continuous monitoring of the actual conditions of the aircraft and its subassembly indicates that the condition of certain components has fallen below a certain critical level.

Aircraft maintenance actions refer to activities that must be performed during aircraft fleet maintenance. The main activities are replacements, repairs and checks. A replacement refers 
to the activity that involves the removal of a failed component from an aircraft or aircraft subassembly and the installation of a serviceable spare component in its place. In order that replacement can be carried out quickly by maintenance crews, an aircraft usually consists of line replaceable units (LRUs). A LRU, which can be quickly replaced in the operational location, is a complete functional unit which consists of a number of shop replaceable units (SRUs). A SRU represents a part of the function of its parent LRU and is removed, replaced and repaired at a back-shop, which is a specified store or workshop where aircraft repair is performed. Repair is one of the most important activities in aircraft maintenance, where failed, repairable components in the aircraft are restored to a working state.

Aircraft checks are PM actions where aircraft structural members, systems and components are systematically examined, inspected and tested to find potential unserviceable conditions. This significantly reduces the risk of operational failures which can cause huge, unpredicted losses. Checks are usually performed in different periodic intervals based on usage or calendar time. Generally, for civil aircraft there are five types of increasingly intensive checks [2]: 'line/transit' check, 'A' check, 'B' check, 'C' check and ' $D$ ' check. Each of the more intensive checks contains all of the tasks from lower order checks. In practice, the intervals, location, cost of manpower and tasks involved in these checks vary by aircraft type, cycle count and the number of hours flown since the previous check. Military aircraft organisations normally have their own specific maintenance check programmes that may not be similar to those of civil operators. For instance, the U.S. Air Force performs a 5-day phase inspection on an individual F-16 aircraft every 400 flying hours [3].

Aircraft fleet maintenance activities are performed at a number of organisations and supported by various resources. Aircraft fleet maintenance resources mainly refer to facilities, spare parts and technicians that support the aircraft maintenance operations. Aircraft maintenance requires various types of maintenance facilities, ranging from hangars to test equipment and tools [4]. An aircraft is an extremely complex system. For instance, there are over 4.5 million parts in a Boeing 747. A spare part is defined as a stocked part in an inventory that is used to replace or repair a failed part [4]. When maintaining an aircraft, spare parts are used to replace components that have failed or need preventive actions in order to quickly restore the aircraft to a condition that allows it to meet operational commitments. Maintenance crews consist of technicians who are responsible for implementing the required maintenance.

Aircraft maintenance activities are organised and classified according to the location in which they take place and the responsibilities of the maintenance crews at these locations. In practice, aircraft organisations have their own identification of maintenance levels. Usually aircraft fleet maintenance is organised in two or three levels. Three-level maintenance includes organisational- (O-level), intermediate- (I-level) and depot-levels (D-level) while two-level maintenance only includes the $\mathrm{O}$ - and D-levels. When an aircraft fails due to component failures, it is usually maintained at the O-level organisation where its failed components are removed and replaced by spares. Removed, failed components are sent to the I-level maintenance organisation for repair or the depot in case of two-level maintenance. Failed components that cannot be repaired at the I-level will be sent to the depot. After repair at I-level or D-level, failed components are returned to the fleet.

In the literature, many models have been established to study aircraft fleet maintenance processes. Interest is mainly focused on the modelling of aircraft PM and CM [5-11], maintenance organisations [12,13] and maintenance resource management [14-20].

Dupuy et al. [5] build a discrete event simulation model based on ARENA to study aircraft PM. Varelies et al. [6] build a simulation model based on I-think dynamic system software to model the life-cycle maintenance of a fleet of aircraft engines. Mattila et al. [7] build a discrete simulation model of the three-level maintenance of a fleet of fighter aircraft in the Finnish Air Force (FiAF). Sheng and Prescott [8] build a hierarchical CPN model for fleet three-level maintenance with cannibalisation, which is a maintenance activity involving the removal of working components from NMC aircraft to restore other NMC aircraft when 
required spares are not available. When studying the workforce-constrained maintenance scheduling problem for a military aircraft fleet, Safaei et al. [9] put the emphasis on the modelling of pre-flight and post-flight inspections. To optimise the repair of aeronautical components, Yang et al. [10] build a CPN model of a detailed aviation component repair process. Feng et al. [11] use a heuristic hybrid game approach to model and optimise the condition-based PM of aircraft fleet. Shell [12] develops a SLAM II simulation model for three-level and two-level maintenance of the Dual Mode Transmitter (DMT, a LRU located in the F-16C/D radar) of a fleet of F-16 C/D aircraft. Wang [13] builds a CPN model to compare the effectiveness of two-level and three-level aircraft maintenance based on fleet availability and maintenance cost. Tang and Zhong [14] build a CPN model of an aircraft-engine assembly procedure considering maintenance resource limitations. Alfredsson [15] builds a mathematical framework to optimise the amount of spares and test equipment of a fleet maintenance system with multi-level repair facilities. Gopalan [16] develops a Rulerian graph model to optimise the location of the aircraft fleet maintenance bases. Gallasch et al. [17] build a CPN model to evaluate and compare the performance of different staffing and scheduling scenarios for maintenance crews in the Australian Army. Sun et al. [18] establisha modelling framework for determining the ordering time and quantity of spares for new aircraft fleets. Qu et al. [19] build a PN model to study maintenance resource scheduling in a military maintenance organisation. Wang et al. [20] build a CPN model of maintenance resource management.

However, existing models of aircraft fleet maintenance only consider a limited number of maintenance factors. Non-maintenance factors such as mission-oriented fleet operation, aircraft failure logic and component failure characteristics, which significantly affect the fleet maintenance performance, are rarely considered. The objective of this paper is to establish a comprehensive framework for modelling aircraft fleet performance, which accounts for all factors related to aircraft fleet maintenance, operation and reliability. The CPN method is selected as the tool to form the framework due to its capability to precisely and concisely graphically represent maintenance processes for large fleets, accurately handling concurrency, parallelism, conflicts, constraints and dependencies among and between maintenance activities and resources, and providing an ideal framework for Monte Carlo simulation.

\section{Analysis of Aircraft Fleet Maintenance Modelling}

To model fleet maintenance, it is necessary to have a good understanding of the related modelling parameters. Aircraft fleet maintenance is a complex process and should not simply involve responding to aircraft failures and performing scheduled maintenance tasks. There are numerous other factors that must be considered, including a variety of maintenance policies and activities, different maintenance organisations and the management of maintenance resources. A comprehensive aircraft fleet maintenance model should therefore be capable of including all of these factors and allowing the impact of maintenance on fleet performance to be assessed using appropriate reliability measures.

Fleet operation and maintenance are highly interrelated. CM tasks in the fleet mainly arrive following fleet operation processes. For example, it is generally assumed that an aircraft and its components can only fail during operation. During peak utility periods, system and component failures are likely to happen more frequently than during off-peak periods, placing a strain on the maintenance organisation and hence meaning that maintenance can have a significant influence on fleet operational performance. For instance, poor maintenance management can lead inoperative aircraft to wait for maintenance for too long a time, meaning that the risk of fleet missions being aborted increases due to the reduced availability of mission capable aircraft. Therefore fleet operational factors should be included in the aircraft fleet maintenance model. Since an aircraft fleet is designed to perform missions, mission-oriented fleet operation processes such as mission scheduling, mission assignment and mission abort events should also be modelled.

The aircraft fleet maintenance model must also consider the aircraft and component failure dynamics. This is important since different combinations of component failures lead aircraft 
to fail in different failure modes, each of which requires different maintenance actions and policies. For example, if some critical component failures cause an aircraft to be non-mission capable, the aircraft will be taken out of service and maintained immediately. If an aircraft with component failures can still perform some of its designed missions, its maintenance may be deferred to a previously-scheduled maintenance event due to critical operational requirements.

Similarly, aircraft components can have multiple failure modes, which require different maintenance activities. It may be possible to repair a failed component at an I-level repair shop or it may be necessary to send the component to a depot, depending on its failure mode. In addition, a component failure process may have more than one phase, for example representing deterioration, leading the modelled component to require multiple component states in contrast to the commonly-modelled binary states, where a component is assumed to either work or fail. More importantly, an aircraft component usually has more than one indenture. An LRU usually consists of a number of SRUs that can be configured with parallel elements to offer redundancy, making the aircraft failure logic more complicated than a simple series configuration.

All in all, a complete model of aircraft fleet maintenance should include multiple factors related to fleet operation, aircraft failure logic, component indenture, component failure characteristics and fleet maintenance that can be divided into four groups:

Fleet-level modelling factors: operation-related fleet factors including mission scheduling, mission assignment and mission-oriented operation.

$>$ Aircraft-level modelling factors: including aircraft failure modes, aircraft failure logic to describe how component failures cause aircraft failure and aircraft state transitions (non-mission capable (NMC) and mission capable (MC)).

> Component-level modelling factors: component structures (LRU and SRU), component failure logic to indicate how lower-indenture failures cause higher indenture component failure and component failure characteristics (failure modes, binary or multiple states).

$>$ Maintenance-level modelling factors: consist of fleet maintenance echelons (threelevel or two-level), aircraft maintenance policies (PM or CM, maintenance task scheduling policy), maintenance resource management (spare inventory control, maintenance crew management, etc.) and details of maintenance activities.

An aircraft fleet maintenance model that incorporates all of these factors can then be used to analyse the impact of an applied maintenance strategy on fleet performance and reliability. The model can be used to calculate measures such as:

- Mission capable rate (assessing fleet reliability by providing the proportion of time that platforms are capable of performing missions in a given time interval);

- Mission abort rate (assessing how often missions must be aborted on the ground or in the air due to platform unreliability);

- Average fleet readiness (assessing the average number of available platforms resulting from the applied maintenance strategy);

- Mean time to repair (assessing the average time taken to restore a NMC aircraft to the MC state);

- Mean time between failures (assessing the average length of time between the failures of an aircraft).

\section{Petri Nets}

First introduced by Carl Adam Petri [21], Petri nets (PN) are powerful, graphical and mathematical tools for modelling complex, dynamic systems [22]. A PN is a directed graph with two types of nodes: places; and transitions, which are connected to one another by directed edges or arcs. Places contain a discrete number of tokens and the distribution of 
tokens within the PN defines its marking, which describes the state of the system modelled at any point in time. Transitions govern the dynamic behaviour of the PN; the system state changes when transitions fire. In order to fire, a transition must first be enabled, which occurs when the number of tokens in each input place is no less than the weight of the arc linking it to the transition. Transitions can be immediate, represented by solid bars, or timed, represented by hollow bars. Immediate transitions fire as soon as they are enabled and timed transitions fire after a delay has elapsed following their becoming enabled.

A timed transition with three input places and three output places is shown in Figure 1 (the place linked by the double-headed arc is both an input and output). The transition is enabled since the number of tokens in each input place is at least as high as the weight of the arc linking it to the transition. After a delay time $t$ has elapsed a number of tokens equivalent to the associated arc weight is removed from each input place and added to each output place.

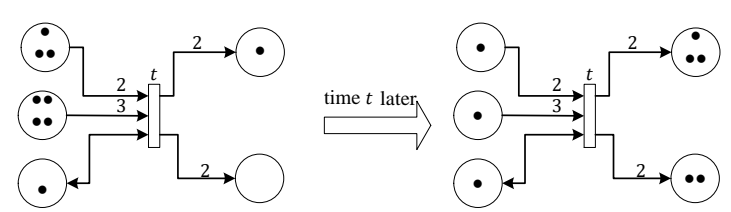

Figure 1. Transition enabling and firing

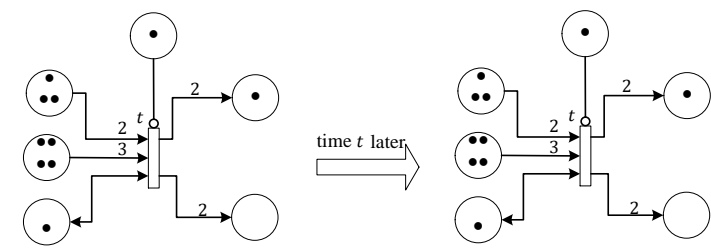

Figure 2. Inhibitor arc prevents transition firing

If a transition is linked to an input place by an inhibitor arc, which has a circle at its head, its firing process is prohibited when the number of tokens in the place is greater than or equal to the weight of the inhibitor arc. Figure 2 shows a transition that is not enabled due to the marking of the place linked to it by an inhibitor arc.

As PN become more complex, the possibility arises for conflicting situations to be encountered [23]. For example, the following may occur:

1. Two or more transitions with different delay times are enabled by the same input place;

2. Two or more transitions with the same delay time (maybe immediate) are enabled by the same input place;

3. An enabled timed transition is disabled while waiting for its delay time to pass.

These conflicts can arise when modelling aircraft fleet operation and maintenance. For example, the first two situations can occur when modelling the scheduling of available maintenance resources if resources are simultaneously requested to perform different maintenance activities. The third situation arises when modelling the termination of component operation processes when an aircraft mission ends.

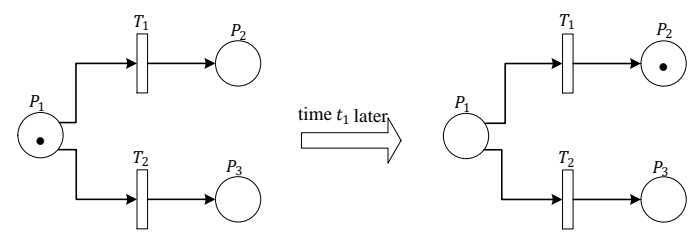

Figure 3. Two timed transitions enabled by the same place

In the first situation, the transitions will fire in an order determined by their firing delay times. The transition whose delay time elapses soonest fires first and switches a constant number (equal to the appropriate arc weight) of tokens from the input place. If, after this, the marking of the input place no longer satisfies the enabling conditions of the other transitions, the other transitions are no longer enabled and will not fire. Figure 3 shows timed transitions $T_{1}$ and $T_{2}$, which are enabled due to the marking of $P_{1}$. If the firing delay times of $T_{1}$ and $T_{2}$ are respectively $t_{1}$ and $t_{2}$ and $t_{1}<t_{2}$, then $T_{1}$ will fire first after a time period $t_{1}$, switching the token from $P_{1}$ to $P_{2}$, which disables the firing of $T_{2}$ since $P_{1}$ is no longer marked.

In the second situation, if the number of tokens in a place exceeds the sum of all arc weights leading from it, all of the enabled transitions will fire simultaneously. If not, the conflict can 
be resolved by assigning a level of precedence to the transitions. This can be determined randomly or based on some discipline such as the priorities of the actions that are represented by the transitions. For instance, the priorities could represent the order in which different maintenance actions are performed when there are insufficient maintenance crews or equipment available to perform all the required actions. The transition with the highest priority fires first and removes the required number of tokens from the place. Subsequent transitions fire in order of their priority until the tokens in the input place are exhausted. Figure 4 shows the firing process of an example PN with two immediate transitions $T_{1}$ and $T_{2}$ being enabled by the same place, where $T_{1}$ is assumed to have higher firing priority than $T_{2}$. When $P_{1}$ is marked, both $T_{1}$ and $T_{2}$ are enabled but $T_{1}$ fires first, immediately removing the token from $P_{1} ; T_{2}$ is no longer enabled. The firing order of the two transitions can also be determined by a probability check [26]. In Figure $4, T_{1}$ fires first with probability $p_{1}$ when both $T_{1}$ and $T_{2}$ are enabled. When $P_{1}$ is marked, a uniform random variable $p$ is sampled in the range from 0 to 1 . If $p \leq p_{1}, T_{1}$ is given a higher firing priority than $T_{2}$. Otherwise, the firing priorities are reversed. This can be used to model the identification of repair shop (I-level or depot) for a failed component where $p_{1}$ represent the probability that the component is repairable at I-level.

The third situation described above can be solved using two main methods: 'memory' transitions and 'memory' tokens [23]. A 'memory' transition 'remembers' the time it was enabled and how much of the delay time remains when its firing process is interrupted and resumes its firing process from the same point when it is enabled again. Alternatively, by attaching a continuously changing 'counter' to each token in an input place, a record of the disabled transition can be assigned to 'memory' tokens instead [23]. In this case, a token is given a counter equal to the transition firing delay, with the counter representing the remaining time that a token will be held by the transition's input place. The token counter decreases continuously while it contributes to the enabling of the transition. The decreasing of the token counter is interrupted when the transition is disabled. In Figure 6, $T_{1}, T_{2}$ and $T_{3}$ have constant firing delays $t_{1}, t_{2}\left(t_{1}<t_{2}\right)$ and $t_{3} . T_{1}$ and $T_{2}$ are both initially enabled by $P_{1}$ and $P_{2} . T_{1}$ fires after time $t_{1}$, moving the token from $P_{1}$ to $P_{3}$, enabling $T_{3}$, which will switch the token from $P_{3}$ to $P_{5}$ after a further time $t_{3}$. The firing process of $T_{2}$ is interrupted because of the marking of its inhibitor place $P_{3}$. In this situation, by using the concept of the 'memory' token, the counter of the token in $P_{2}$ has decreased to $t_{2}-t_{1}$ and will remain at this value until $P_{3}$ is unmarked. After $T_{3}$ fires, $T_{2}$ is enabled again and its previous firing process will be resumed. $T_{2}$ will then fire after a time $t_{2}-t_{1}$, which removes the token from $P_{2}$ and adds it to $P_{4}$.
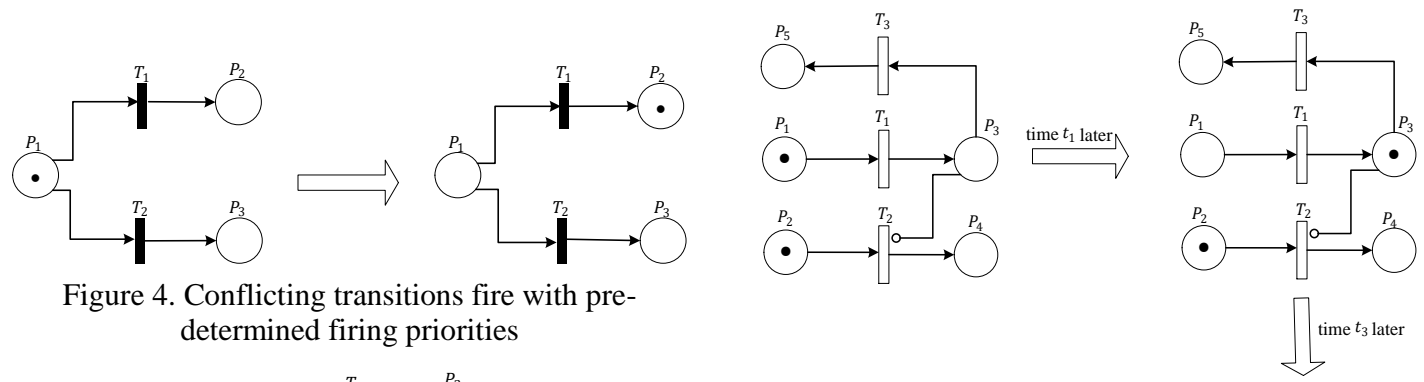
determined firing priorities
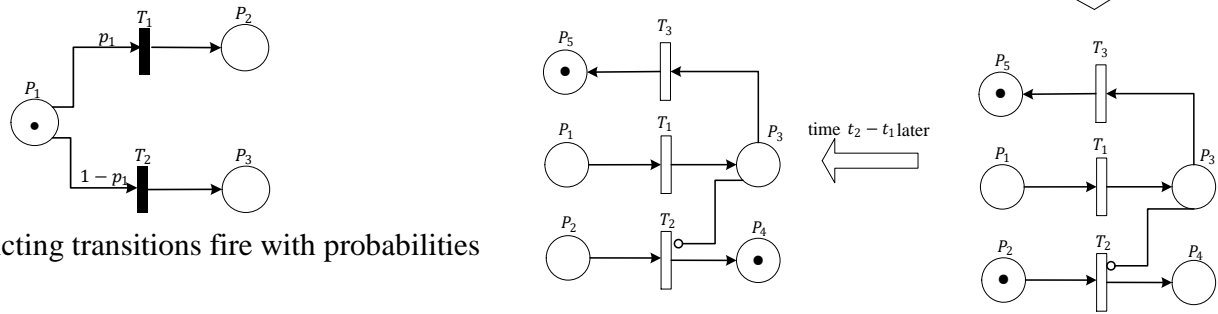

Figure 6. Firing process with memory token

The priorities described above make 'memory' tokens potentially useful for modelling aircraft operation processes. By using a timed transition to represent the failure process of a component, the value of the token counter can be used to represent the remaining working life 
(time to failure) of the component if it can only fail during operation. The token counter decreases when the component is operational, a process which stops when the component is not operational. The timed transition fires when the counter reaches 0 , indicating that its remaining life has declined to 0 and that it has failed.

Created by Jensen and Kristensen [24], coloured Petri nets (CPN) extend PN by allowing the behaviour of complex systems, especially those that are concurrent, to be concisely modelled. Compared with PN, tokens in CPN are distinguished by token colours. The colour is a label that can represent different data types, such as Boolean, integer or character strings. Each place has a colour set, which holds the possible token colours of a specified type. Each arc has an arc expression, which determines the number of tokens of specific colours that are removed from or added to the linked place when a transition fires. Each transition has a guard, which is a Boolean expression that represents a constraint on its enabling policy.

A transition is enabled only if the marking of each input place satisfies the associated input arc expression and its guard evaluates to be true. Transitions can fire tokens of different colours in different ways and the colour of a token can change after it passes through a transition as decided by a specific modelling condition. Figure 7 shows an instance of transition enabling and firing for a CPN.

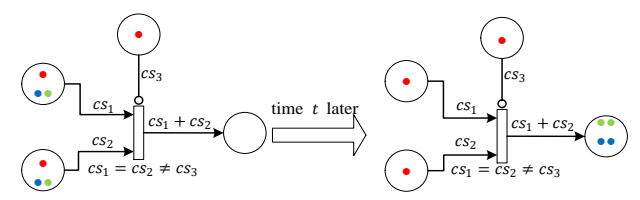

Figure 7. Transition enabling and firing of CPN

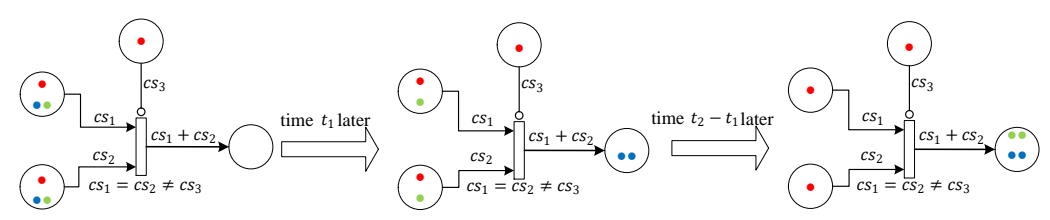

Figure 8. Transition fires tokens with different switching time

The timed transition in Figure 7 has three input places and one output place, all of which have the same colour set $C S=\{$ green, red, blue $\} . c s_{1}, c s_{2}$ and $c s_{3}$ are colour variables that belong to $C S$. The switching of blue and green tokens in the input places is enabled but the red token in the input place linked by the inhibitor arc prevents that firing of red tokens. After a delay time $t$ has elapsed the transition moves the blue and green tokens from the input places to the output place.

In Figure 7, different coloured tokens have the same delay time in. Generally, tokens with different colours will have different delay times, for example modelling the times to failure of various components. Figure 8 shows the effects of colours on the firing of transitions. The delay times of blue and green tokens are $t_{1}$ and $t_{2}\left(t_{1}<t_{2}\right)$ respectively. After the delay time $t_{1}$, the blue tokens move to the output place. After a further delay time $t_{2}-t_{1}$, the transition moves the green tokens from the input places to the output places.

\section{CPN Modelling of Aircraft Fleet Maintenance}

As discussed in Section 2, aircraft fleet maintenance processes are affected by four main factors that relate to aircraft failure logic, component failure characteristics, detailed maintenance activities and maintenance management and fleet operation. A comprehensive model, which is able to consider all these factors, predict fleet performance and evaluate fleet maintenance strategies will be of great benefit in aiding fleet maintenance decision making.

Four groups of CPN models are built to represent the processes of fleet operation, aircraft failures, component failures and fleet maintenance. These proposed CPN are initially designed for military aircraft fleets, which are usually deployed at one or more bases in order to perform missions. A typical mission might be a transportation task, a daily training flight, a long-distance bombing raid or an air combat task. For instance, when a training flight is called 
for a fighter aircraft fleet, the fleet manager will check to see if there are enough idle, MC aircraft to be assigned to the mission. If not, the training flight is cancelled and a ground abort happens. Otherwise, the required number of $\mathrm{MC}$ aircraft take off from the base where they are deployed to perform the required training course following the planned flying route. Aircraft return to base upon mission completion or if they experience a failure. An aircraft is assumed to consist of a number of repairable components which can fail during operation and can contribute to the failure of the aircraft. The aircraft failures considered in this paper are non-catastrophic, meaning that an aircraft cannot perform a mission required of it due to component failures, as opposed to catastrophic, which would lead to loss of the aircraft. Maintenance of a failed aircraft is carried out through the replacement of its failed components using available resources. Failed components are repaired and then added to the fleet spare supply system. Restored aircraft are returned to the fleet to perform missions. MC (mission capable) aircraft are periodically inspected in order to avoid unexpected failures.

The CPN models proposed here are designed to provide a CPN framework that can be used to describe the factors affecting aircraft fleet maintenance. Though oriented to military aircraft fleets, the modelling framework can also be used to study other fleets of vehicles, such as civil aircraft, tanks and helicopters, which have similar maintenance processes to military aircraft. The framework is flexible so that it can be modified and extended through the inclusion or substitution of new modules if other operational or maintenance actions must be considered, without necessarily amending existing modules.

\subsection{CPN Modelling of Fleet Operation}

The mission-oriented fleet operation can be divided into three parts: mission scheduling, mission assignment and mission operation, which respectively correspond to determining when to perform the required missions, deciding which aircraft should perform scheduled missions and the detailed operating processes that assigned aircraft should perform.

\subsubsection{CPN Modelling of Mission Scheduling}

Fleet mission scheduling refers to identifying the start time of each mission. In CPN, prior to assignment, different missions can be represented by different coloured tokens held in a specified place. Mission scheduling can then be achieved by a timed transition that assigns firing delays to tokens in its input place, representing the mission start times. Missions might be pre-determined and start at regular, specified times or could depend on factors beyond the control of the fleet manager, in which case mission assignment will be more dynamic.

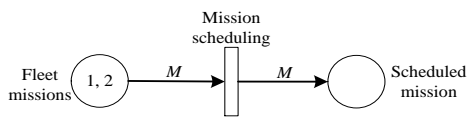

Figure 9. CPN of mission scheduling

Figure 9 shows a CPN model of mission scheduling for an example fleet with two required missions that are respectively represented by indices 1 and 2, with labels to describe the meanings of its places and transitions. The colour variable $M$ represents an arbitrary mission. The transition 'Mission scheduling' is enabled and will respectively assign each of token 1 and 2 with a firing delay. These firing delays might be pre-determined or sampled from a known probability distribution, depending on the nature of the mission assignment and how this affects their start times. The firing policy of tokens with different firing delays was introduced in Section 3.

\subsubsection{CPN Modelling of Mission Assignment}

When a mission is called, the required number of MC aircraft will be assigned to perform it by the fleet manager. An assigned aircraft can be FMC (fully mission capable) or PMC (partly mission capable) so long as it satisfies the MEL (minimum equipment list) for the scheduled mission, which identifies the components that must be operative in order for the aircraft to be capable of performing the mission. An aircraft is FMC when it can perform all possible designated missions, while it is PMC when it is able to perform at least one of its designated missions. A FMC aircraft could have a higher assignment priority than a PMC 
aircraft. However, this may vary between fleets and will be decided by fleet managers. A mission may be cancelled if the number of available aircraft does not satisfy the minimal requirement for the mission. This type of mission cancellation is called a ground abort event.

As shown in Figure 10, the mission assignment event can be represented by an immediate transition with an inhibitor arc. An aircraft can be assigned to perform a scheduled mission only if it is mission capable and idle, which means it is not performing other missions. Therefore, the transition 'Mission assignment' is enabled when a mission $M$ is scheduled to be performed and there is an idle MC aircraft (represented by $A$ ). Once enabled it fires immediately, assigning idle MC aircraft to the mission. When the number of tokens in the place 'Assigned aircraft' is equal to $N M_{M}$, the number of aircraft required to perform the mission, the transition is inhibited since enough aircraft have been assigned to carry out the mission.

When considering FMC and PMC aircraft, information regarding the condition of individual aircraft components should be included in the mission assignment model. If FMC aircraft are assumed to have higher assignment priorities than PMC aircraft, then this can be modelled using transition firing priorities. A modified model of mission assignment that considers FMC and PMC aircraft and uses transition firing priority is shown in Figure 11. The assignment process is represented by two immediate transitions: 'Assign FMC aircraft' and 'Assign PMC aircraft', with the former having a higher priority than the latter. FMC aircraft are assigned in preference to PMC aircraft until the supply of FMC aircraft is exhausted, at which point the transition 'Assign PMC aircraft' will fire if enabled, a situation that occurs if there are PMC aircraft whose operative components meet the MEL of the scheduled mission.

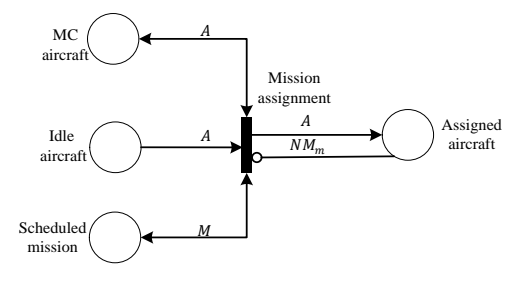

Figure 10. CPN of mission assignment

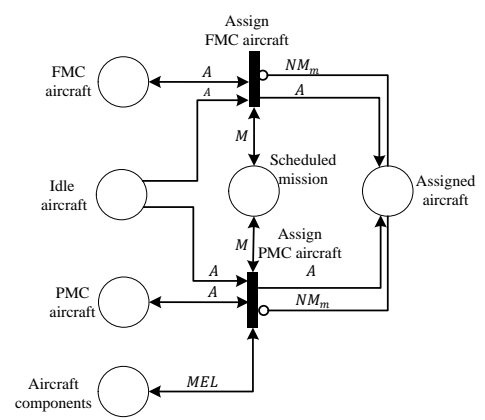

Figure 11. CPN of mission assignment using transition priority

A fleet mission must usually start within a certain time of it being scheduled. A scheduled mission may be cancelled if the assigned $\mathrm{MC}$ aircraft cannot reach the mission requirements before the assignment time window of the mission closes. If a mission is ground aborted in this way, aircraft that have been assigned to the mission will be released and made available to perform other missions. Otherwise, the scheduled mission will start once enough aircraft are assigned. This process is represented by the CPN module shown in Figure 12. For a mission, $M$, the immediate transition 'Mission starts' is enabled and the transition 'Mission ground abort' is inhibited if $N M_{M}$ aircraft have been assigned to perform the mission, represented by tokens in the place 'Assigned aircraft'. Once enabled, 'Mission starts' fires immediately, switching the token from the 'scheduled mission' to the place 'Mission start'. Otherwise the transition 'Mission ground abort' is enabled and will fire after a time delay representing the maximum assignment time for $M$. When 'Mission ground abort' fires, the token with colour $M$ in the place 'Scheduled mission' will be moved to the place 'Cancelled mission', stating that a mission ground abort event happens. This enables the transition 
'Release assigned aircraft', which will release the aircraft that have already been assigned to the cancelled mission.

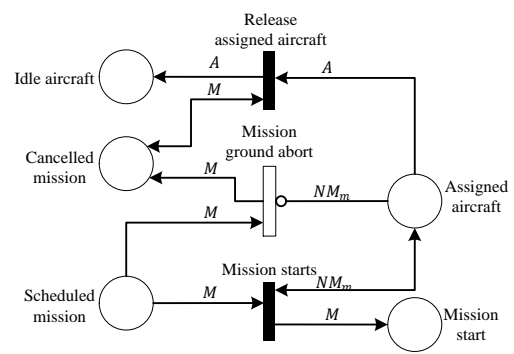

Figure 12. CPN of mission ground abort

\subsubsection{CPN Modelling of Mission Operation}

When a mission starts, assigned aircraft take off to perform it and land after operating for a number of flying hours determined according to the mission. Note that the required number of flying hours for different aircraft in the same mission can vary. The varying requirement for different aircraft can be represented by a timed transition, which assigns different firing delays to different coloured tokens. As shown in Figure 13, aircraft begin their operations when the mission to which they are assigned starts, which is governed by the transition 'Aircraft operation starts'. Once a scheduled mission starts, 'Aircraft operation starts' fires immediately, moving all tokens from the place 'Assigned aircraft' to 'Operating Aircraft'. The operating duration for an assigned aircraft, represented by a coloured token in 'Operating aircraft', is represented by the firing delay that is assigned to the token by the transition 'Aircraft operating'. A mission ends when all assigned aircraft land, which can be represented by the immediate transition 'Mission ends' with an inhibitor arc, shown in Figure 14. For the mission $M$, the transition 'Mission ends' is enabled only if there are no remaining assigned, operational aircraft.

If an aircraft becomes NMC while performing a mission, an air abort event happens and the aircraft is taken out of service and sent to the maintenance shop for immediate maintenance. The aircraft failures considered in this paper refer to non-catastrophic failures, which mean that an operating aircraft cannot perform the required mission due to component failures, not catastrophic failures which would lead to loss of the aircraft. The non-catastrophic failures are represented by an immediate transition 'Mission air abort' shown in Figure 15, which is enabled when places 'Operating aircraft' and 'NMC Aircraft' contain tokens of the same colour, which represent the same aircraft. Once enabled, 'Mission air abort' fires immediately, removing the token representing the failed aircraft from 'Operating aircraft' and adding it to the aircraft maintenance queue.

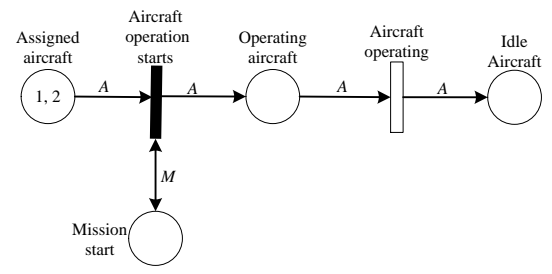

Figure 13. CPN of aircraft operating

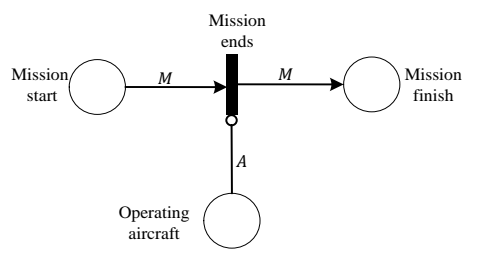

Figure 14. CPN of mission ends

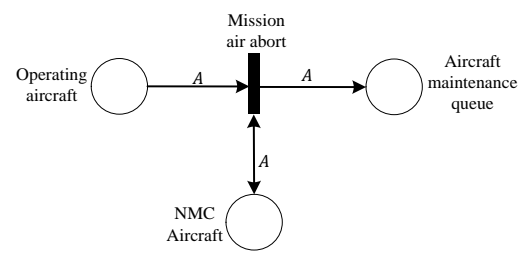

Figure 15. CPN of mission air abort 


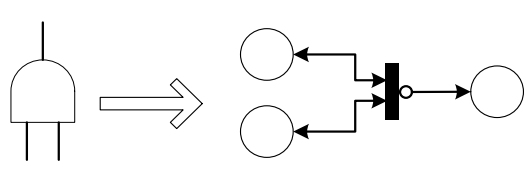

(a)

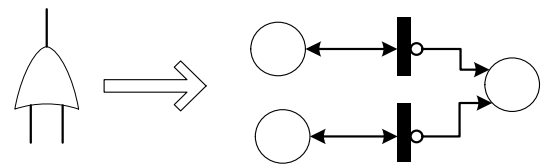

(b)

Figure 16. PN of AND and OR gates [22]

\subsection{CPN Modelling of Aircraft Failure}

The non-catastrophic failure of an aircraft occurs when some of its components fail during operation. An aircraft can have numerous failure modes, each of which is caused by a different combination of component failures. The causes of aircraft failure modes can be represented by fault trees (FTs) and in order to be considered in the CPN modelling framework, the FT logic must be converted to a CPN format. Chew [22] introduced a method of building an uncoloured PN model for a given FT. In his method, each event is converted to a place. As shown in Figure 16, an AND gate is converted to a single immediate transition and an OR gate is converted to a number of immediate transitions equivalent to how many input events it has. In each case, the input places represent the event inputs to the modelled gate. The double-headed arcs are designed to keep a record of the state of input events during the PN simulation. The inhibitor arcs prevent an infinite number of immediate transition switches from taking place and stop transitions from firing if the event modelled by the output place has already occurred and that place is therefore already marked [22].

Since this conversion of a FT to a PN has no colour, the use of it to model fleets of identical aircraft will require an inefficient replication of the entire PN, with one PN being required for each failure mode for each aircraft.

However, by employing colours, arc expressions and transition guards, identical failure modes from any number of identical aircraft can be modelled using a single CPN. In a CPN all basic events are represented by the single place 'Component failure' with each basic event represented by a different coloured token. All non-basic events are represented by a place each. Thus the CPN model of an AND gate is an immediate transition whose basic event inputs are represented by a single place. Figures 18 and 19 respectively show the converted CPN for the AND gates $G_{5}$ and $G_{2}$ of the example FT shown in Figure 17. The AND gate $G_{5}$ is represented by the immediate transition ' $G_{5}$ ' while basic events $C_{9}$ and $C_{10}$ are represented by the place 'Component failure' and the place ' $S_{4}$ ' describes the intermediate event $S_{4}$. The transition is enabled when components $C_{9}$ and $C_{10}$ of the same aircraft fail during a mission. Once enabled it fires immediately, creating a token whose colour represents the intermediate event $S_{4}$ of the aircraft in the output place. The CPN for the AND gate $G_{2}$ is similar, with its basic event inputs represented by a single place and its intermediate event input, $S_{3}$, being represented as an input to the transition by the place created to model it.

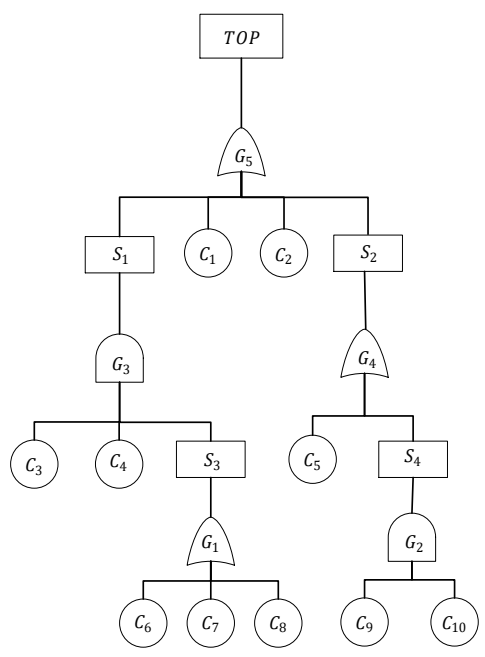

Figure 17. Example aircraft fault tree

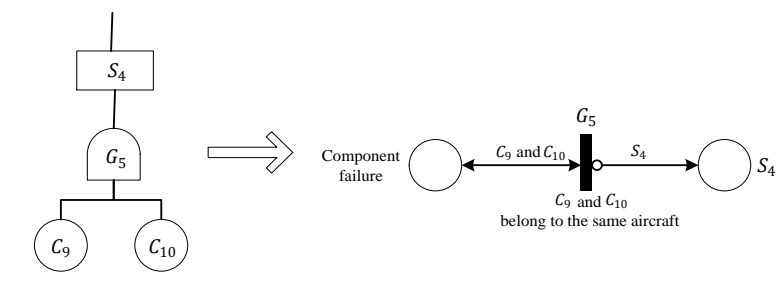

Figure 18. CPN of AND gate G5
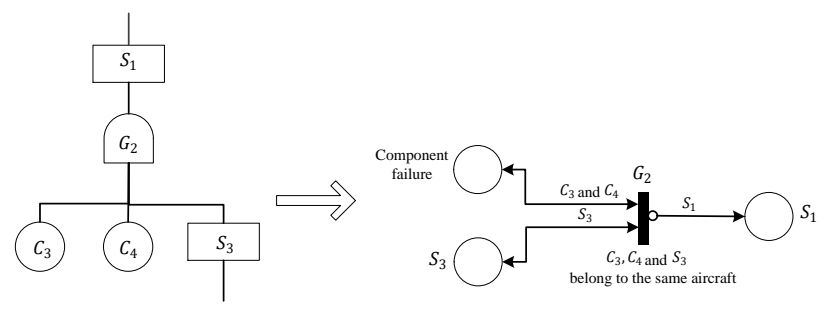

Figure 19. CPN of AND gate G2 
For an OR gate, the corresponding CPN consists of a number of immediate transitions whose input places represent the OR gate's event inputs. Figure 20 shows the converted CPN for the OR gate $G_{1}$. The gate is converted to three immediate transitions $G_{1}(1)-G_{1}(3)$, for which the output place represents an aircraft failure event (since the output of $G_{1}$ is the top event). The transition $G_{1}(1)$ is enabled when either of components $C_{1}$ or $C_{2}$ fail. $G_{1}(2)$ and $G_{1}(3)$ are enabled when the intermediate events $S_{1}$ and $S_{2}$ occur respectively. As soon as one of the three transitions has fired, and a token representing the failed aircraft has been placed in 'Aircraft failure', then all three transitions are disabled to ensure that only one token representing the failed aircraft can appear in 'Aircraft failure'. Using these rules for converting FT to CPN, a CPN for the example FT shown in Figure 17 can be constructed; the result is shown in Figure 21.

Figure 22 models the aircraft state transitions. The state of an aircraft changes from MC to NMC when some of its critical components fail and vice versa following restoration. These processes are respectively represented by transitions 'Aircraft failed' and 'Aircraft maintained'.

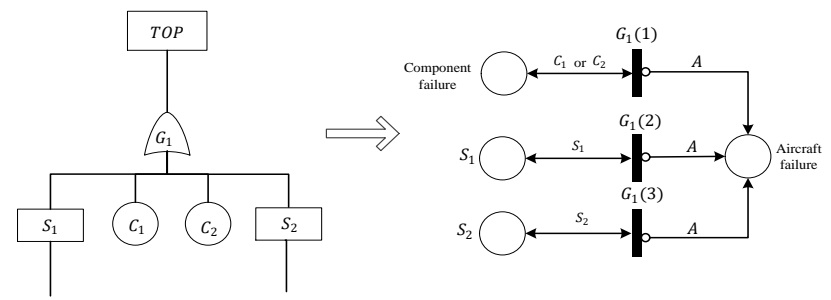

Figure 20. CPN of an example OR gate

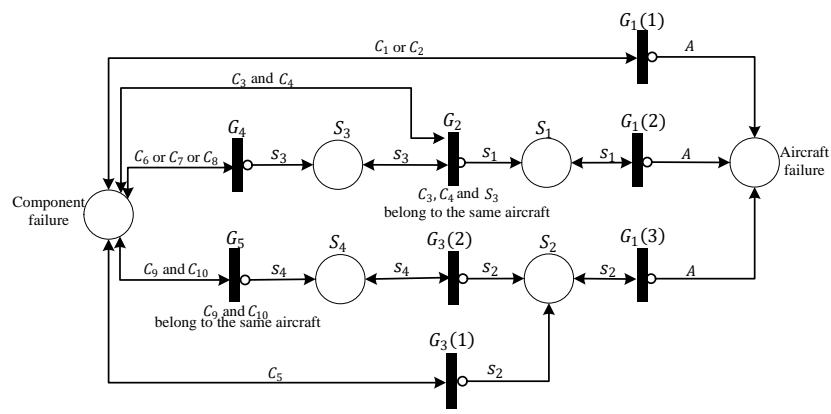

Figure 21. CPN of the example FT

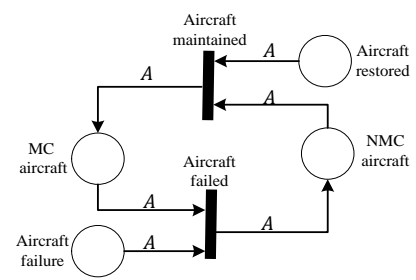

Figure 22. CPN of aircraft state change

\subsection{CPN Modelling of Component Failure}

Modelling aircraft component failures forms the basis of aircraft fleet maintenance modelling. Component failures not only induce aircraft failure events but also lead to the requirement for specific component maintenance tasks. Components could be considered to have binary states, in which case they could either work or fail, or to have multiple states, which could be used to model component degradation of the type that might be observed in a condition monitoring process as part of a preventive maintenance scheme. Component indentures must also be considered since aircraft, in common with other complex and expensive platforms such as ships, usually consist of multi-indentured components. Redundant components are often employed in aircraft in an attempt to increase reliability. The above situations can introduce dependencies, e.g. component failure dependencies that arise due to components experiencing an increased load due to the failure of other components or due to active and standby redundancies, where the failure rates of components change according to whether they are in 
operation or on standby for other components. Component repair dependencies can also arise due to component indenture. Whilst these dependencies could not be modelled using a technique such as Fault Tree Analysis, CPN models can be developed to analyse each of these situations, as shown in the following subsections.

\subsubsection{Binary State Components}

In binary-state theory, a component is assumed to have two states: 0 (working) and 1 (failed). To model such a component, Chew [22] build an uncoloured PN model with a timed transition as shown in Figure 23. Places ' 0 ' and ' 1 ' respectively represent the working and failed states of an individual component. Every binary-state aircraft component will have its own PN model in which one uncoloured token is placed in either place ' 0 ' or ' 1 '. The transition is enabled when place ' 0 ' is marked. Once enabled a time delay will be sampled from a known probability distribution, which is defined according to the failure characteristics of the modelled component.

It is possible to represent all binary-state components on an aircraft using a single CPN, as shown in Figure 24. The place 'Component working' represents all working binary-state components of all aircraft in the fleet studied. For a component $C$, it can fail only when the aircraft $A$ to which it is fitted is performing a mission. The transition 'component fails' is enabled if places 'Component working' and 'Operating aircraft' respectively contain coloured tokens $C$ and $A$ that satisfy the transition guard ' $C$ belongs to $A$ '.

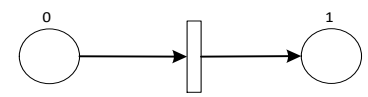

Figure 23. PN model of binary-state component failure [22]

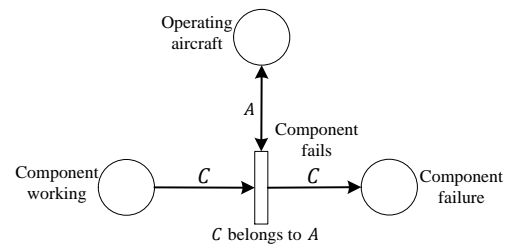

Figure 24. CPN model of binary-state component failure

If the component $C$ fails before the aircraft $A$ finishes performing the current mission ('Operating aircraft' is still marked with token $A$ when the sampled firing delay of token $C$ runs out), the transition will switch the token $C$ from 'Component working' to 'Component failure', indicating that component $C$ fails. It is assumed that component $C$ only fails during operation meaning that it cannot fail once the mission has ended either because of component failures leading to the aircraft becoming NMC or because the current mission is complete. This means that the failure process can be interrupted. To resume the interrupted failure process when the aircraft is operating again, the component condition (remaining working life) at the break point must be memorised. The 'memory' token, introduced in Section 3, is employed to achieve this action in this paper. The component remaining life is represented by a child variable of the component colour $C$. The variable is initialised by sampling from a known probability distribution and decreases when the transition 'Component fails' is enabled by the related component. The component failure process is interrupted when the related firing process is disabled. When resuming a component failure process, the transition will assign a new firing delay to the component that is equal to its remaining working life.

An aircraft component can fail in different modes due, for example, to fatigue, corrosion or overload. Different component failure modes can induce different aircraft failure modes and require different maintenance actions. For example, a fuel system valve may fail closed leading to an interruption of the fuel supply and a subsequent loss of power, or fail open, meaning the fuel supply cannot be cut and engine thrust not reduced. Figure 25 shows the CPN model for a component $C$ with $N_{C F M}$ failure modes represented by $1, \ldots, N_{C F M}$. The conflicting transitions representing failure in each mode are enabled at the same time and will each assign a firing delay to the working component $C$ that represents the expected time to fail in a particular mode. As introduced in Section 3, the transition with the smallest firing 
delay fires first, removing token $C$ from 'Component working' and adding a token to 'Component failure,' identifying the failed component and its related failure mode.

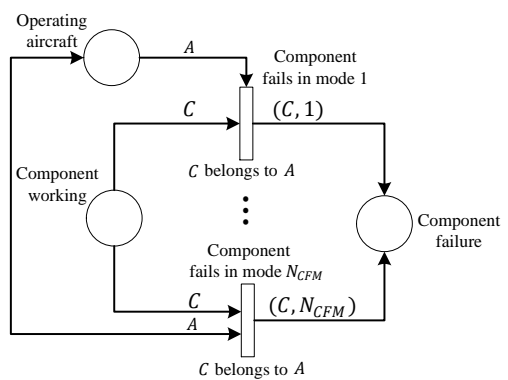

Figure 25. CPN of multiple component failure modes

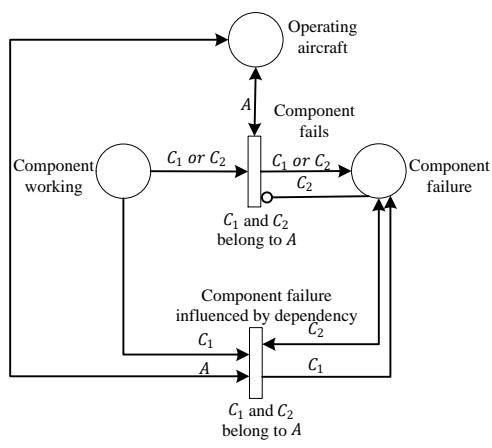

Figure 26. CPN of dependent component failures

The CPN models shown in Figures 24 and 25 are built based on the assumption that component failures are independent. However, dependency may exist between the failure processes of different components. The failure of a component may accelerate or even directly result in the failure of other components. For instance, a computer processor will overheat and fail much sooner if the fan fails. With the assumption that the failure of component $C_{1}$ is accelerated if component $C_{2}$ fails, Figure 26 shows the CPN model for dependent components. The transition 'Component fails' is almost the same as that in Figure 24 except for the extra inhibitor arc. If component $C_{2}$ of aircraft $A$ fails first during operation, the normal firing process of component $C_{1}$ of aircraft $A$ by the transition 'Component fails' is interrupted by the inhibitor arc. Instead, the transition 'Component failure influenced by dependency' is enabled and a new time delay is assigned to the firing of the token with colour $C_{1}$. The time delay is sampled based on the expected remaining life of the component $C_{1}$ and the new probability distribution of the altered failure process. The sampling policy transfers the expected remaining life of the component to an equivalent expected remaining life in the new failure probability distribution [23]. If the remaining life (in working hours) of component $C_{1}$ is $c a$ when component $C_{2}$ fails and $F_{1}$ and $F_{2}$ are CDFs of the ordinary and accelerated failure probabilities respectively, the equivalent remaining life $c a_{1}$ can be represented by:

$$
c a_{1}=F_{2}^{-1}\left[F_{1}(c a)\right]
$$

\subsubsection{Multiple State Components}

Sometimes binary state theory oversimplifies real situations where the conditions of components can be classified into a range of levels from perfect function to complete failure. For instance, as introduced by Gurler and Kaya [25], railroad tracks, telecommunication systems and infrastructure systems fail with multiple states. Multi-state theory should be applied to modelling aircraft component failures because of PM actions, especially related to condition-based maintenance.

When modelling multiple-state components, the first step is to identify all the possible component states. A mathematical description of multi-state component theory is a state array or a set with finite variables such as $(0, \ldots, N)$ where 0 and $N$ respectively signify the perfect working state and the complete failure state. When the states are identified, the CPN model can be built by assigning each state its own place and using a timed transition to represent the change from one state to the next.

As an example, Figure 27 shows the CPN model for a component $C$ with 3 states: working, PM due and failed. The transition 'Component needs PM' represents the degradation of the working component to the condition where preventive replacement is necessary. The process from PM due to failed is described by the transition 'PM component fails'. The enabling and firing polices of the two transitions are similar to those of the transition 'Component fails' in Figure 24. 


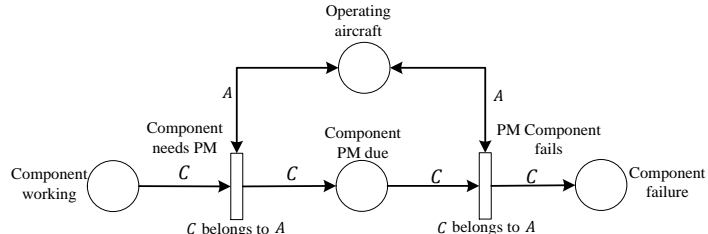

Figure 27. CPN of multi-state components under a condition-based PM policy

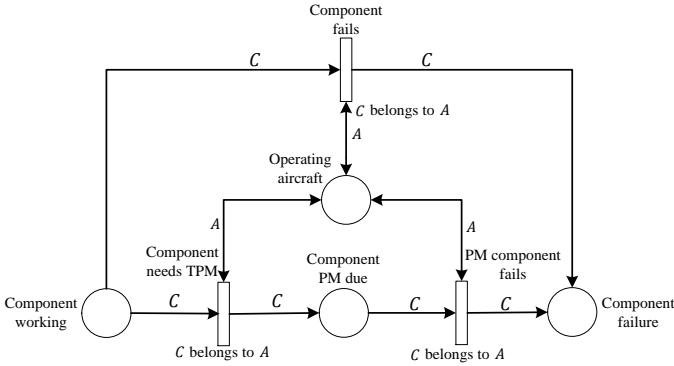

Figure 28. CPN of multi-state components under a time-based PM policy

While under time-based PM, components are preventively replaced at pre-determined intervals. There is a probability that a component fails before its usage reaches the PM threshold. This means that the state of the component may directly change from working to failed. The CPN model shown in Figure 27 is not suitable for such components. The modified model which considers time-based PM is shown in Figure 28. When a component $C$ in an operational aircraft $A$ enables the transition 'Component fails,' the remaining life of the component in the working state decreases. The timed transition 'Component needs TPM' with fixed firing delay represents the transition from the working state to the PM due state. The fixed firing delay of 'Component needs TPM' represents the PM threshold (in cumulative working hours) of the component $C$. If the fixed firing delay runs out before the remaining life of the component declines to 0 , transition 'Component needs TPM' fires, moving the token $C$ to the place 'Component PM due'. This enables the transition 'PM component fails', which models the remaining component working life, given its usage when PM was identified as being due.

\subsubsection{Component Indenture}

Line replaceable units, LRUs, allow the rapid replacement of multiple components. A LRU can be viewed as a multiple indentured component that is made up of shop-replaceable units, SRUs, configured as necessary to provide the required levels of functional redundancy. A SRU can be replaced at a repair shop when it causes the LRU to fail. A SRU may also be multiple indentured and consist of a number of lower-indenture components.

The failure of a higher-indenture component can be viewed as an immediate event that is induced by the failure of its lower-indenture components. The failure processes of lowestlevel components can be modelled by a timed transition as shown in Figure 24. The failure process of higher-level components can be represented by immediate transitions that take inputs from places which describe the failures of their child components.

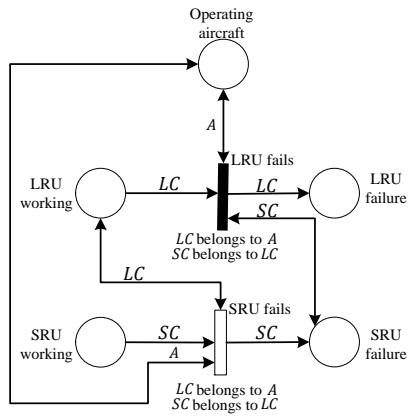

Figure 29. CPN of two-indenture component

Figure 29 shows the CPN model for an example two-indenture LRU whose child SRUs are connected in series. The timed transition 'SRU fails' and immediate transition 'LRU fails' represent the failure processes of the SRUs and LRU respectively. Colour variables $L C$ and $S C$ respectively represent a LRU and a SRU. The enabling policy of the transition 'SRU fails' is similar to that of the transition 'Component fails' in Figure 24 except for the requirement that a SRU is able to work only if the LRU to which it is fitted is working. This requirement 
represents the fact that a LRU may be a functional unit with elements of the overall function provided by each SRU. When a SRU SC fails, transition 'LRU fails' is enabled and fires immediately, moving the token with colour $L C$, representing the parent LRU of $S C$, from 'LRU working' to 'LRU failure'. This models the failure of LRU $L C$ on aircraft $A$.

\subsubsection{Component Redundancy}

Component redundancy is usually introduced to expensive, safety-critical platforms such as aircraft in order to improve platform reliability and availability and to reduce the number of unexpected failures. When an online, functioning component fails during operation, a redundant component will be brought into operation to act in its place and ensure the system can continue to operate. Redundant components can be active or standby. Active-redundancy components simultaneously start to operate alongside their related functioning components. Standby-redundancy components can be cold, warm or hot. Cold standby components do not operate and suffer failures until they are brought into operation. Warm standby components work continuously during aircraft operation but fail with a lower probability when on standby than when operational, while hot-standby components provide active redundancies [22]. In the standby case, redundant components are sequentially switched into operation when active, functioning components fail. The switching may be performed either automatically, where sensors continuously monitor online components and activate redundancies accordingly, or manually, where an operator is responsible for bringing redundant components online [22]. Analysis of redundancies should also include switching failures since these will prevent redundant components from becoming operational when required. Automatic switching may happen anytime due to sensor failures, while manual errors are likely to occur with some probability during switching [22]. Component dependencies arise due to: the operational states (active or standby) of redundant components being determined by the state (working or failed) of the related online components; whether switching is successful after component failure; and the acceleration of cold- and warm-standby component failures when they are switched online.

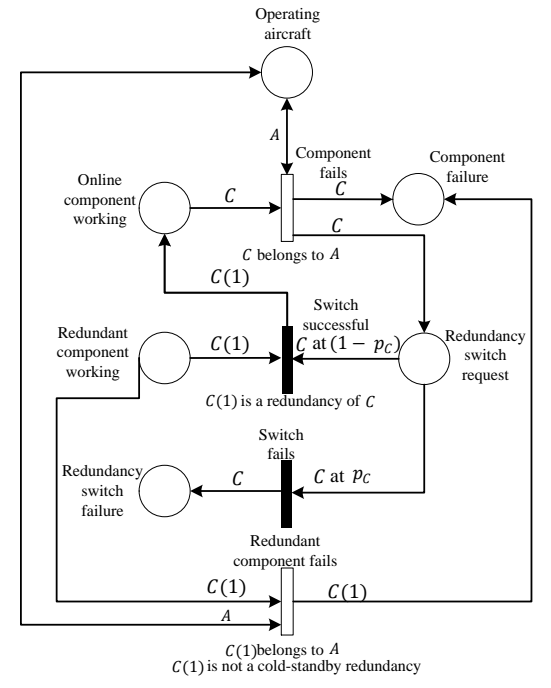

Figure 30. CPN of component redundancy with manual switching

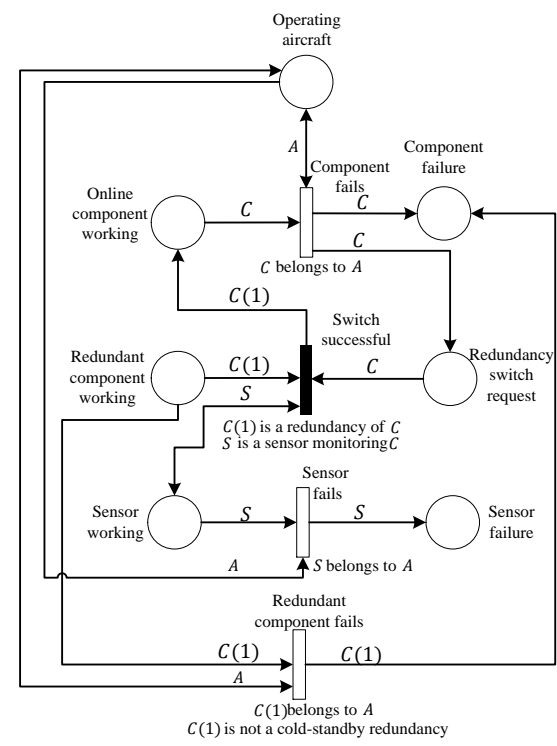

Figure 31. CPN of component redundancy with sensor failures

Since active redundant components function alongside primary components, no switching process is required. The failure of an active redundant component can hence be modelled by the CPN model shown in Figure 24. For standby redundancy, Figure 30 represents an example CPN model considering a manual switching error. For a component $C$, the probability of human error during switching is assumed to be $p_{C}$. When an online component $C$ fails (the transition 'Component fails' fires), a redundancy switch request will be called as a token is added to the place 'Redundancy switch request'. This enables the transitions 'Switch successful' and 'Switch fails'. A random number, $r(0 \leq r \leq 1)$, is sampled from a uniform 
distribution. If $r>p_{\mathrm{C}}$, the transition 'Switch successful' will fire and move the token $C(1)$, the redundant component of the failed component $C$, from 'Redundant components working' to 'Online component working'. Otherwise, the transition 'Switch fails' will fire and a manual redundancy switch failure happens. If the redundant component $C(1)$ is not cold standby, the standby component could fail before the online component fails. This process is represented by the transition 'Redundant component fails' which samples a failure time for a warm or hot standby component.

Since a component is less likely to fail when on warm standby than when operational, its failure process will be altered when brought into operation. This is governed by the transition 'Component fails', which samples a firing delay and assigns it to the related token in the place 'Online component working', representing the equivalent expected online remaining life of the switched component, based on the operational failure probability distribution and the time it has spent on standby. The detailed sampling policy of the transition 'component fails' is similar to that of the transition 'Component failure influenced by dependency' in Figure 26 which was introduced in Section 4.3.1. As a component on hot standby experiences the same failure processes as its online component, the transition 'component fails' assigns a firing delay equivalent to its remaining life on hot standby when the component is switched into operation.

Figure 31 shows a CPN model of redundancy with automatic switching. The place 'Sensor working' represents the sensors that monitor the failure of online components being in a working state. As opposed to manual switching, when the standby component $C(1)$ for online component $C$ must be switched when $C$ fails, the transition 'Switch successful' can be enabled only if the sensor $S$ is working (place 'Sensor working' contains a token with colour $S$ ) since it must detect the change in condition of $C$. The failure processes of the sensors are represented by the transition 'Sensor fails'. As introduced in the case of manual switch, when the switch of a warm-standby component happens, a firing delay which represents the expected online remaining life will be assigned to the related component by the transition 'Component fails'.

\subsection{CPN Modelling of Fleet Maintenance}

Aircraft fleet maintenance can be performed at three levels: O-level, I-level (optional) and Dlevel, using corrective and scheduled maintenance actions. The O-level maintenance organisation is mainly responsible for the restoration of inoperative aircraft to the working state through the replacement of failed components, or those requiring preventive replacement, using spares. After removal, consumable components are discarded and repairable components are sent to the I-level maintenance organisation for repair. If a component is declared to be non-repairable at the I-level maintenance organisation, it is then sent to the depot for repair. Repaired components are used to replenish the spare supply chain.

\subsubsection{O-level Maintenance Model}

The major maintenance tasks of the O-level maintenance organisation are the corrective and preventive maintenance of NMC aircraft. When an aircraft fails, it is immediately taken out of service and added to the aircraft maintenance queue which holds all the NMC aircraft that must be restored. Maintenance crews first remove the failed components from the aircraft and then fill the 'holes' with spare components. To prevent mission air abort events, aircraft are periodically inspected and any components that need to be are replaced under a preventive maintenance strategy. Therefore, the major O-level maintenance activity is component replacement.

Failed components of a NMC aircraft can be replaced only if the aircraft has been placed into the aircraft maintenance queue. Figure 32 shows a CPN which identifies the component replacement tasks for the O-level maintenance organisation. The transition 'Set component replacement queue' is enabled and fires immediately when a token with colour $A$ is added to the place 'Aircraft maintenance queue', moving tokens representing the failed components of aircraft $A$ from the place 'Component failure' to the place 'Component replacement queue'. 


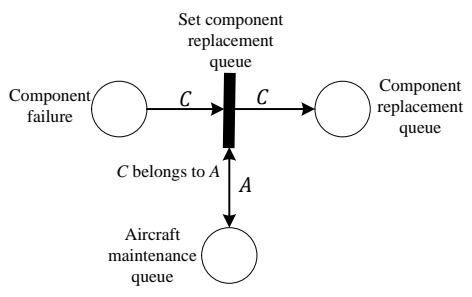

Figure 32. CPN of set component replacement queue

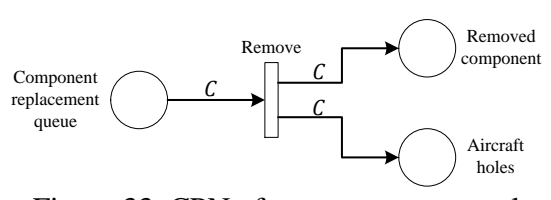

Figure 33. CPN of component removal

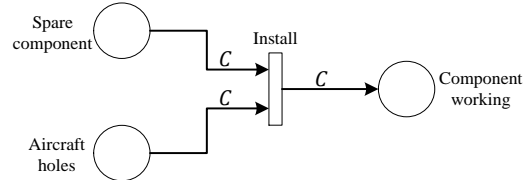

Figure 34. CPN of component installation

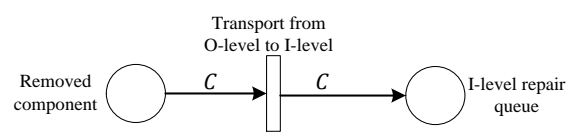

Figure 35. CPN of transport from O-level to I-level

In CPN, a maintenance activity can be modelled as a timed transition whose firing delays represent the time taken to complete the activity. A component replacement action consists of two sequential activities: removal and installation. Figure 33 shows the CPN model for component removal. The transition 'Remove' represents the process of removing failed components. For a token in 'Component replacement queue', the firing delay assigned to this token represents the time taken to remove the failed component represented by the token. The CPN model for the installation of spare components is shown in Figure 34. When a failed component has been removed and a spare is ready, the transition is enabled and will fire after a period of time representing the installation time. The removal/installation time could be modelled as being constant or sampled from a known distribution, determined from an analysis of the actual maintenance processes. After removal, failed components will be transported to the I-level maintenance queue for repair. This process is represented by the CPN model in Figure 35. The firing delay associated to the transition 'Transport from O-level to I-level' represents the actual time taken to transport failed components from the O-level maintenance organisation to the I-level maintenance organisation.

Aircraft check intervals are usually usage-based. A usage-based aircraft check can be modelled as an immediate transition that is driven by aircraft flight hours. A time-varying parameter is added to the aircraft colour $A$ that represents the number of hours that the aircraft needs to fly before its next check; this allows the CPN model for an aircraft ' $A$ ' check shown in Figure 36 to be built. The initial value of the parameter is equal to the aircraft 'A' check interval (represented by $T_{A C}$ ). The parameter decreases during aircraft operation (the transition 'Aircraft operating' in Figure 13 is enabled by the aircraft). When this parameter reaches 0 for a MC, idle aircraft $A$ (both places 'MC aircraft' and 'Idle aircraft' have a token with colour $A$ ), the transition 'Aircraft check' is enabled and fires immediately, when a token with colour $A$ will be switched from 'MC aircraft' to 'Aircraft maintenance queue', with the decreasing parameter reset to $T_{A C}$, meaning that a new check cycle will start for the aircraft. This enables the transition 'Set PM component replacement queue', which immediately places any components with the PM due state on the aircraft undergoing the check into the component replacement queue.

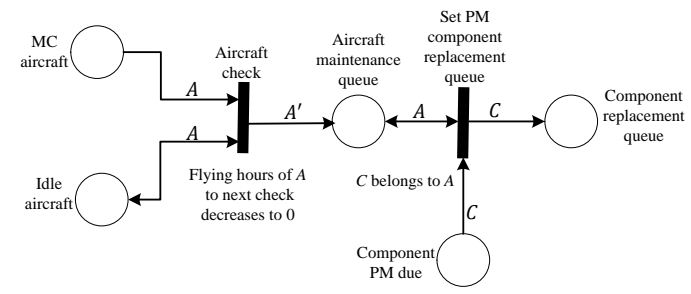

Figure 36. CPN of aircraft checks

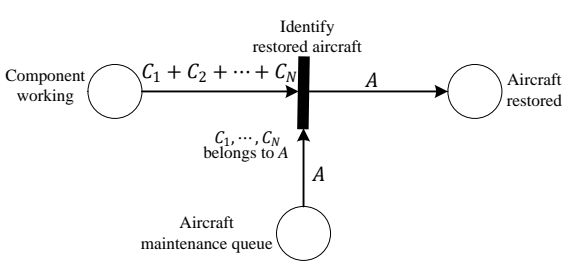

Figure 37. CPN of aircraft restored 
Similarly, other aircraft fleet checks ('B', ' $\mathrm{C}$ ' and ' $\mathrm{D}$ ') can be modelled through adding new parameters to the aircraft colour $A$. The value of a new parameter is initially the interval of the related aircraft check and will continuously decrease as the related aircraft is performing missions. Note that each of the more intensive checks usually contains all of the tasks from lower order checks. When any parameter of a MC, idle aircraft decreases to 0 , the transition 'Aircraft check' fires immediately, sending the aircraft for the related check and resetting the parameter and those relating to the lower order checks of the aircraft to the corresponding check intervals.

A NMC aircraft that is undergoing maintenance is restored when all its components are working. The CPN model in Figure 37 identifies the restored aircraft. Assume an aircraft consists of $N$ components, labelled $C_{1}, C_{2}, \ldots, C_{N}$. The transition 'Identify restored aircraft' is enabled when there are $N$ different components in the place 'Component working' which belong to the same aircraft. Once enabled, 'Identify restored aircraft' fires immediately, moving the token representing the restored aircraft from 'Aircraft maintenance queue' to 'Aircraft restored'.

\subsubsection{I-level Maintenance Model}

A removed component is first sent to the I-level maintenance organisation to check whether it can be repaired there or not. If not, the component will be classified as NRTS (not repairable this station) and sent to the depot. Otherwise I-level maintenance crews carry out the necessary repairs to restore it to the working condition. In the developed CPN model it has been assumed that a component is repairable at the I-level repair shop with a certain probability, which depends on the component type. If $p_{\mathrm{C}}$ represents the probability that component $C$ is repairable at I-level, a CPN such as that given in Figure 38 models repair shop allocation. The two transitions are enabled when a failed component $C$ arrives at the Ilevel maintenance shop. A number, $r$, is randomly sampled from a uniform distribution. If $r \leq$ $p_{\mathrm{C}}$, the transition 'Component repairable at I-level' fires and moves the token $C$ from 'I-level repair queue' to 'Component I-level repairable'. Otherwise, the transition 'Component NRTS at I-level' fires and the failed component is stated non-repairable at I-level and sent to the depot by the transition 'Transport from I-level to D-level' in Figure 39. If the modelled fleet employs two-level maintenance, $p_{C}$ is set to zero for all component types, effectively excluding the I-level maintenance model.

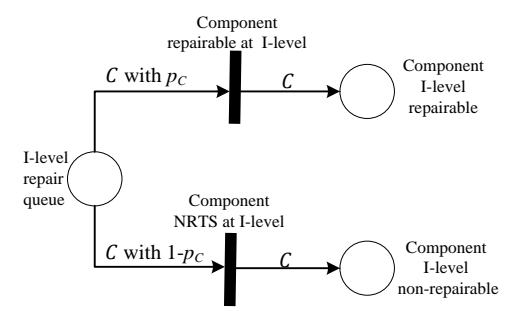

Figure 38. CPN of component repair shop allocation

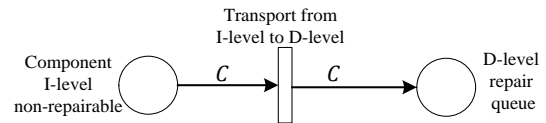

Figure 39. CPN of transport from I-level to D-level

Figure 40 shows the CPN model of I-level component repair. The influence of repair is reflected in the modelled expected working life of repaired components. Under perfect repair ('as good as new'), the expected working life of a repaired component $C$ is newly sampled from the known probability distribution relating to the component failure time. Using an additional variable to memorise the age (in cumulative working hours) of the component since its last repair, under minimal repair ('as bad as old') the new component working life is obtained by sampled from the distribution based on the component age, while under imperfect repair the new component working life is sampled according to a younger age between 0 and the age immediately prior to repair. The representation of the repair impact is achieved by the timed transition 'I-level repair' which changes the time-varying parameters of tokens in the 
'Component I-level repairable' when they are passed to it by 'I-level repaired component'. The firing delay assigned to a token $C$ by transition 'I-level repair' represents the actual time taken to repair component $C$ in the I-level maintenance organisation. After repair at the I-level maintenance organisation, components will be sent back to the O-level maintenance organisation, which form the new spare inventory. This process is represented by the CPN model in Figure 41.

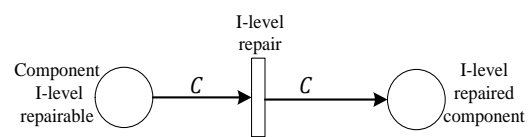

Figure 40. CPN model of I-level component repair

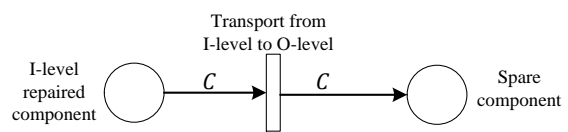

Figure 41. CPN of transport from I-level to O-level

\subsubsection{D-level Maintenance Model}

It is assumed that failed, repairable components can always be repaired at the depot. The Dlevel maintenance model describes the repairs of failed components in the depot, which are similar to those in the I-level repair shop. The repair time of similar components may be different at I-level and D-level repair shops due to possible discrepancies in repair capabilities for reasons such as number and type of repair tools and maintenance crew skills. The CPN model for depot repair is shown in Figure 42. After repair at the depot, the transition 'Transport from D-level to O-level' in Figure 43 is responsible for sending new spares back to the O-level maintenance organisation from the depot.

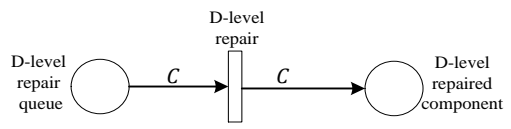

Figure 42. CPN of D-level component repair

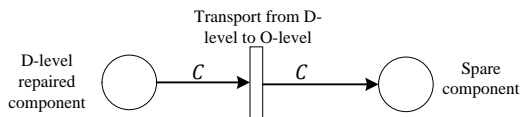

Figure 43. CPN of transport from D-level to O-level

\subsubsection{Manpower Limitations}

Since there is a finite number of maintenance crews, there is a probability that a maintenance task will have to be delayed because maintenance crews are occupied. Figure 44 shows how to modify the CPN model for the removal of failed components when there is a limited number of maintenance crews. $n_{\mathrm{C}}$ represents the number of maintenance crews required to remove component $C$. The place 'O-level maintenance crews' represents the number of idle maintenance crews in the O-level maintenance organisation. When a failed component $C$ must be removed, the removal work can only begin if there are $n_{\mathrm{C}}$ idle O-level maintenance crews. If so, the transition 'Removal starts' fires immediately and O-level crews start to remove the component. After the component is removed (transition 'Remove' fires), the previously occupied $n_{\mathrm{C}}$ crews are idle again and can be assigned to perform other maintenance tasks. A similar modified component installation model accounting for manpower limitations is shown in Figure 45.

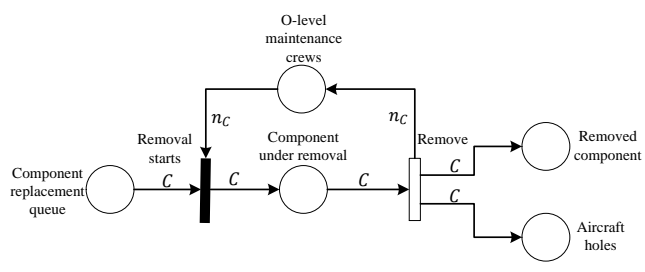

Figure 44. CPN of component removal with manpower limitation

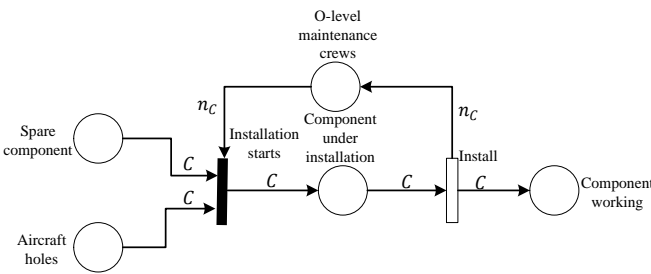

Figure 45. CPN of component installation with manpower limitation

Further, there could be a conflict when multiple maintenance actions are called at the same time but there are not enough idle crews to perform them all. In such a situation, it is necessary to properly allocate and schedule the maintenance crews according to maintenance objectives. This can be achieved by prioritising the maintenance tasks. This is achieved in the CPN model using the priority transitions introduced in Section 3. For example, the scheduling of component removal and installation can be achieved by setting the firing priorities of transitions 'Removal starts' and 'Installation starts'. 


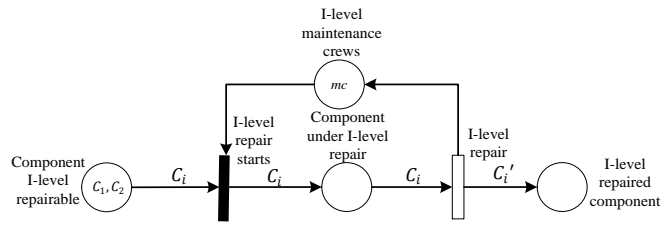

Figure 46. CPN of I-level component repair with manpower limitation

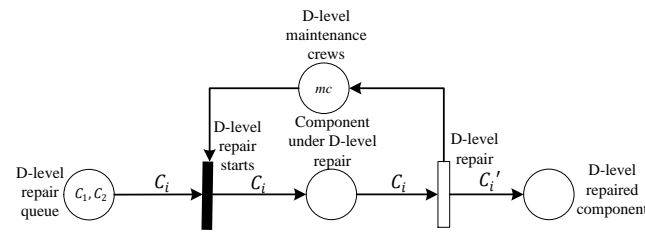

Figure 47. CPN of D-level component repair with manpower limitation

These conflicts do not only arise for different maintenance activities but also in different tasks relating to the same maintenance action, such as the repairs of different types of components. The repairs of failed components for which there are no spares in stock may need to be performed first if a NMC aircraft is waiting for that spare. This can be solved by assigning different priorities to different coloured tokens in the input place representing the queue of components to be repaired. Figure 46 shows the I-level component repair CPN model that is modified to allow maintenance crew constraints to be considered. If two components $C_{1}$ and $C_{2}$ are placed into the I-level repair queue but there is only one idle crew (represented by $m c$ ), the maintenance manager should decide which one to repair first. If the repair of $C_{1}$ is more urgent, the transition 'I-level repair starts' will first fire and move the token $C_{1}$ from the place 'Component I-level repairable' to the place 'Component under I-level repair', removing the token $m c$ from 'I-level maintenance crews'. The repair of $C_{2}$ must wait until an idle maintenance crew is available. Similarly, the modified D-level component repair module which accounts for manpower limitations is shown in Figure 47.

\section{CPN Model Framework}

The CPN model of aircraft fleet maintenance is made up of four modules (explained in detail in Sections 4.1 to 4.4 respectively):

- Fleet module - models mission-oriented aircraft fleet operation;

- Aircraft module - models aircraft failures and changes of aircraft state;

- Component module - models component failures;

- Maintenance module - models the three-level aircraft fleet maintenance processes.

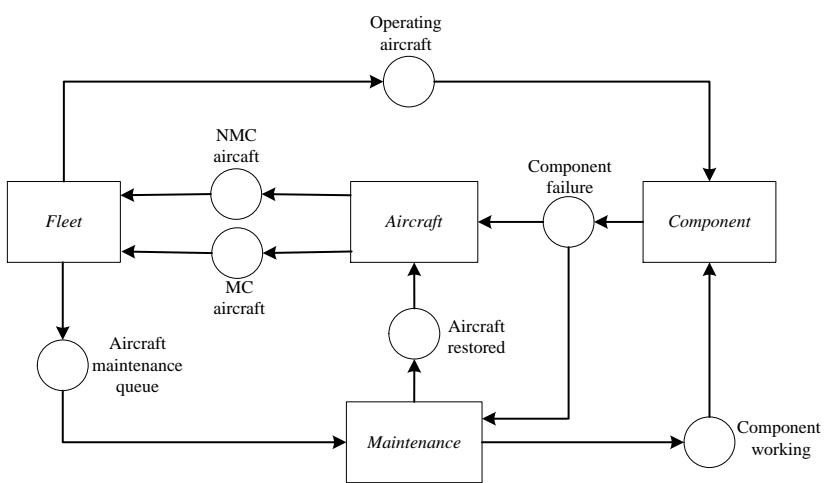

Figure 48. Connections between CPN modules

Figure 48 shows the connections between the four modules. The fleet module is responsible for scheduling, assigning and performing the missions required of aircraft in the fleet. When a mission is called, the fleet module assigns MC aircraft (represented by tokens in the place ' $\mathrm{MC}$ aircraft') to perform it, placing tokens representing the selected $\mathrm{MC}$ aircraft into the place 'Operating aircraft'. This activates the component module, which outputs component failures to the aircraft module through the place 'Component failure'. If component failures cause an aircraft to become NMC, the aircraft module, in turn, places the aircraft failure event into the fleet module through the place 'NMC aircraft'. The fleet module then takes the NMC aircraft out of service and sends it to the aircraft maintenance queue immediately. The maintenance module governs the restoration of NMC aircraft by replacing their failed components by spares. After all failed components are replaced, restored aircraft and their working components are respectively output to the aircraft module and the component module through places 'Aircraft restored' and 'component working' by the maintenance 
module. The aircraft module changes the state of the restored aircraft from NMC to MC, signifying that they are available to be assigned to perform missions by the fleet module again.

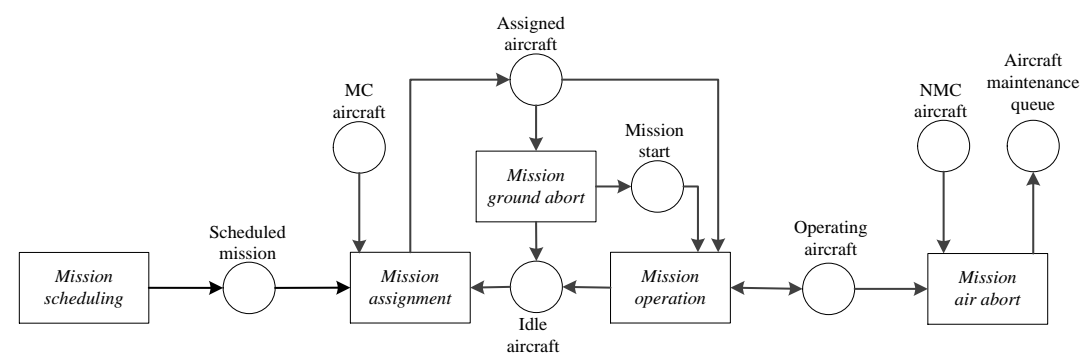

Figure 49. Fleet module structure

The structure of the fleet module is shown in Figure 49. The fleet module consists of five submodules: mission scheduling, mission assignment, mission ground abort, mission operation and mission air abort. When a mission is scheduled, the mission scheduling sub-module outputs the mission to the mission assignment sub-module, which assigns MC, idle aircraft (tokens with same colour in places 'MC aircraft' and 'Idle aircraft' ) to perform the mission. If the required number of aircraft (represented by tokens in the place 'Assigned aircraft') have been assigned to the mission before its assignment time window closes, the scheduled mission starts and a token is added to the place 'Mission start' by the mission ground abort submodule. Otherwise, the scheduled mission is cancelled and the mission ground abort submodule returns the pre-assigned aircraft to the mission assignment sub-module, stating that they can be assigned to other missions. The mission operation sub-module governs the operation of the assigned aircraft, adding the operating aircraft to the place 'Operating aircraft' and returning them to the place 'Idle aircraft' after the mission ends. If an operating aircraft becomes NMC during operation, the mission air abort sub-module will add it to the aircraft maintenance queue.

Figure 50 shows the aircraft module, which has two sub-modules: aircraft failure and aircraft state change. When an aircraft fails due to component failures, the aircraft sub-module outputs the aircraft failure to the aircraft state change sub-module, where the state of the failed aircraft is changed from $\mathrm{MC}$ to $\mathrm{NMC}$ (the related token is removed from the place 'MC aircraft' to the place 'NMC aircraft'). After restoration, the aircraft state change sub-module will reset the state of the NMC aircraft to $\mathrm{MC}$ again.

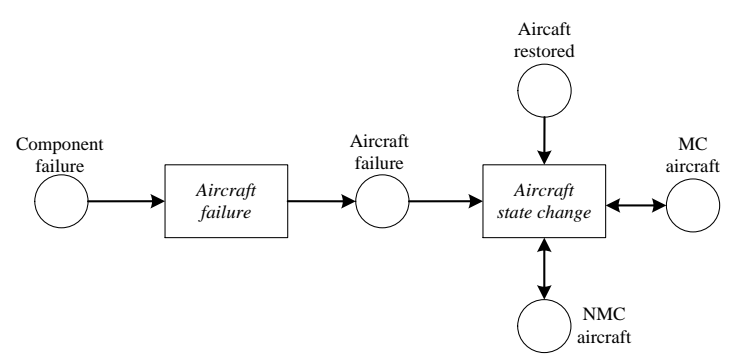

Figure 50. Aircraft module structure

The component module is responsible for modelling the component failure events which happen on operating aircraft. A number of component modules that differ according to the component state, failure dependency, indenture and redundancy have been introduced in Section 4.3. Fleet managers can select the appropriate module based on their own component structure.

The maintenance module, having three sub-modules, is shown in Figure 51. NMC aircraft in the aircraft maintenance queue (tokens in 'Aircraft maintenance queue') are maintained in the O-level maintenance sub-module, where their failed components (tokens in 'Component failure') are replaced by spares (tokens in 'Spare component'). When the restoration of an aircraft is finished, the O-level maintenance sub-module will remove the aircraft from the place 'Aircraft maintenance queue' and add it to the place 'Aircraft restored', showing its replaced components in the place 'Component working'. Removed, failed components at O- 
level are first added to the I-level repair queue so that repair can be attempted in the I-level maintenance sub-module. If they are found to be repairable at I-level, maintenance crews will repair them and send them back to I-level through the place 'spare component'. Otherwise, they will be sent to the depot to be repaired in the D-level maintenance sub-module, which will return them to the O-level maintenance sub-module as spares after repair. The details of the O-level maintenance, I-level maintenance and D-level maintenance sub-modules are shown in Figures 52-54 respectively.

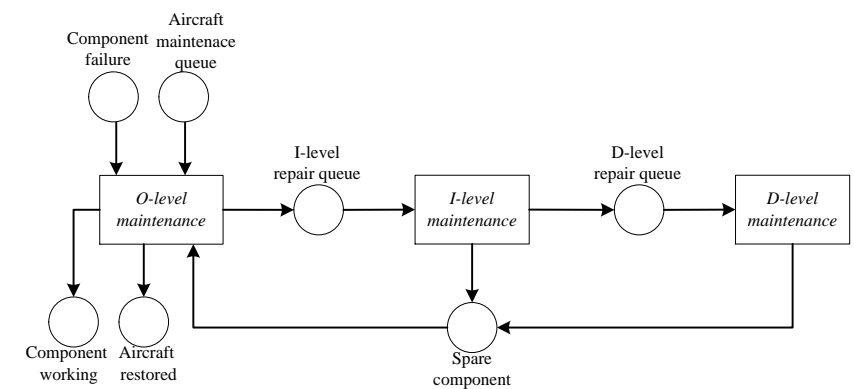

Figure 51. Maintenance module structure

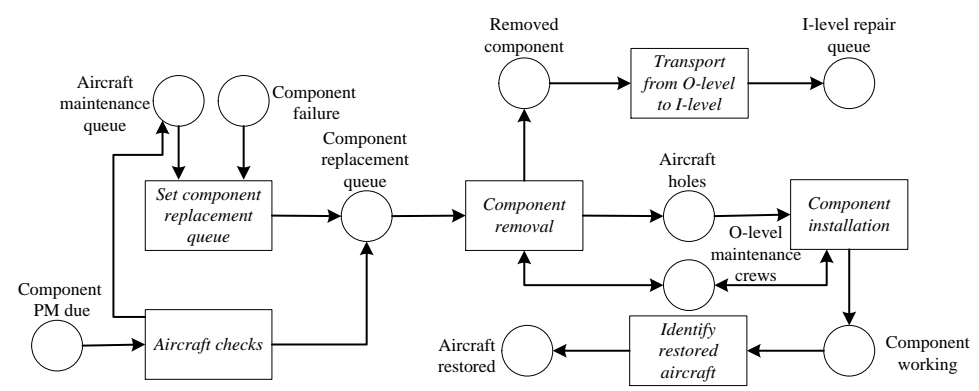

Figure 52. O-level maintenance sub-module structure

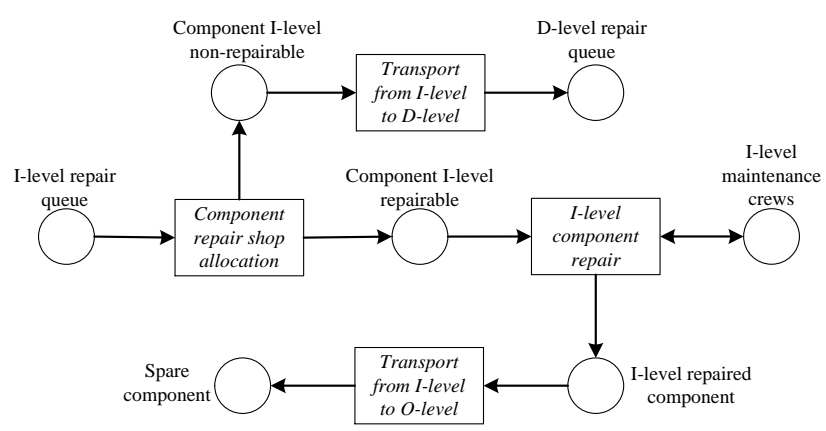

Figure 53. I-level maintenance sub-module structure

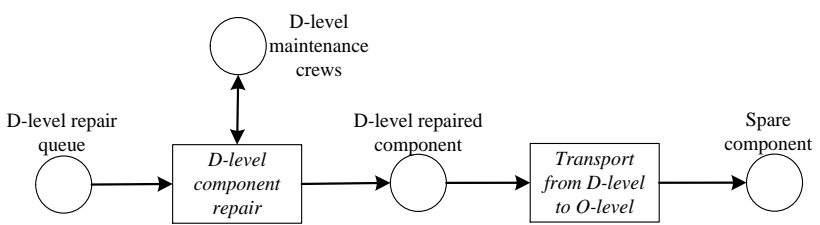

Figure 54. D-level maintenance sub-module structure

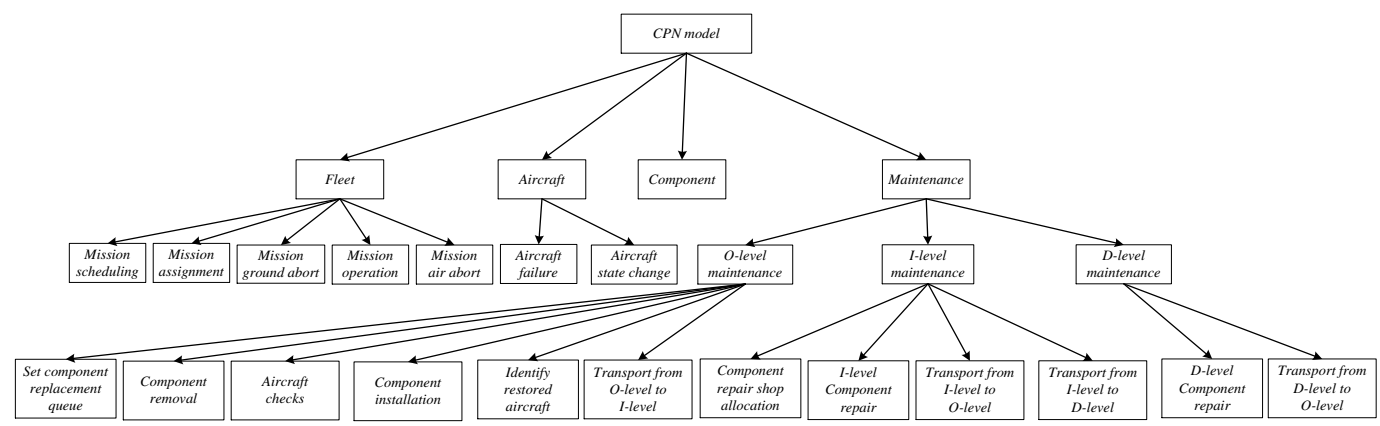

Figure 55. CPN model framework 
The overall CPN framework is shown in Figure 55. When applying the CPN model to a specific fleet, first model users need to define the token colours, place colour sets, arc expressions and transition guards for each module. Secondly, fleet parameters such as the number of aircraft within the fleet, the number of components within an aircraft, spare inventory, component removal/installation time and component repair time at I-level and Dlevel must be input to the CPN model by setting the related place markings and firing delays. Tables 1 and 2 respectively list places and transitions that must be initialised and the associated input parameters. In addition, the required number of MC aircraft to perform a scheduled mission and the I-level repair probability of failed components are respectively governed by the arc expressions of the inhibitor arc linking the place 'Assigned aircraft' to the transition 'Mission assignment' in the mission assignment sub-module and the input arc from the place 'I-level repair queue' to the transition 'Component repairable at I-level' in the component repair shop allocation sub-module.

Table 1. Input parameters represented by place marking

\begin{tabular}{|c|c|c|}
\hline Place & Module & Input parameter \\
\hline Fleet missions & $\begin{array}{l}\text { Mission } \\
\text { scheduling }\end{array}$ & The total number of required missions \\
\hline MC aircraft & $\begin{array}{l}\text { Aircraft state } \\
\text { change }\end{array}$ & The total number and indices of platforms in the fleet \\
\hline Component working & Component & The total number and indices of components in platforms \\
\hline Spare component & $\begin{array}{l}\text { Component } \\
\text { installation }\end{array}$ & The number of spares for each type of component \\
\hline $\begin{array}{l}\text { O-level maintenance } \\
\text { crews }\end{array}$ & $\begin{array}{c}\text { O-level } \\
\text { maintenance }\end{array}$ & $\begin{array}{l}\text { The number of maintenance crews at the O-level } \\
\text { maintenance organisation }\end{array}$ \\
\hline $\begin{array}{l}\text { I-level maintenance } \\
\text { crews }\end{array}$ & $\begin{array}{c}\text { I-level } \\
\text { maintenance }\end{array}$ & $\begin{array}{l}\text { The number of maintenance crews at the I-level } \\
\text { maintenance organisation }\end{array}$ \\
\hline $\begin{array}{l}\text { D-level maintenance } \\
\text { crews }\end{array}$ & $\begin{array}{l}\text { D-level } \\
\text { maintenance }\end{array}$ & $\begin{array}{l}\text { The number of maintenance crews at the D-level } \\
\text { maintenance organisation }\end{array}$ \\
\hline
\end{tabular}

Table 2. Input parameters represented by transition firing delays

\begin{tabular}{|c|c|c|}
\hline Transition & Module & The start time of each mission \\
\hline $\begin{array}{c}\text { Mission scheduling } \\
\text { Mission ground } \\
\text { abort }\end{array}$ & Mission scheduling & The assignment time window of each mission \\
\hline Aircraft operating & Mission operation & The length of each mission \\
\hline Component fails & Component failure & Component failure time \\
\hline Remove & Component removal & Removal time of failed components \\
\hline Install & Component installation & Installation time of spare components \\
\hline I-level repair & I-level component repair & Component repair time at I-level \\
\hline D-level repair & D-level component repair & The transportation time from O-level to I-level \\
\hline $\begin{array}{c}\text { Transport from O- } \\
\text { level to I-level }\end{array}$ & $\begin{array}{c}\text { Transport from O-level to } \\
\text { I-level }\end{array}$ & The transportation time from I-level to O-level \\
\hline $\begin{array}{c}\text { Transport from I- } \\
\text { level to D-level }\end{array}$ & $\begin{array}{c}\text { Transport from I-level to } \\
\text { D-level }\end{array}$ & The transportation time from I-level to O-level \\
\hline $\begin{array}{c}\text { Transport from I- } \\
\text { level to O-level }\end{array}$ & $\begin{array}{c}\text { Transport from I-level to } \\
\text { O-level }\end{array}$ & The transportation time from D-level to O-level \\
\hline $\begin{array}{c}\text { Transport from D- } \\
\text { level to O-level }\end{array}$ & $\begin{array}{c}\text { Transport from D-level to } \\
\text { O-level }\end{array}$ & \\
\hline
\end{tabular}

\section{Model Analysis}

Due to the stochastic nature of aircraft fleet operation and maintenance processes, calculating fleet performance through analytical solution of the CPN model is impossible. After the characteristics of all places, transitions and arcs of the CPN model have been identified for the modelled fleet, the initialised model can be used as a framework for a Monte Carlo simulation analysis of the modelled fleet.

Monte Carlo simulation uses repeated random sampling and statistical analysis to obtain the required system performance [29]. Performing a Monte Carlo simulation for an aircraft fleet needs a computer model of the fleet operation, which must have a set of rules to govern the responses to all possible events relating to the fleet operation and maintenance processes such as component failures, repairs, removals and installations. The construction of a computer 
model relies on a logical representation of the operation of the studied fleet, identifying all the events that can occur, all the activities that can be performed and the correspondence between the events and activities [27]. In this case, the presented CPN model of aircraft fleet maintenance provides a graphical description of the fleet operation. This can then form a framework for the establishment of the computer model.

In order to obtain the required fleet performance measures, the computer model then simulates the occurrence of fleet events and corresponding activities for a suitable period of time, for example the system lifetime or the length of a few missions [27]. During the simulations, the times relating to events and activities such as the component failure and repair times can be obtained by randomly sampling from the associated probability distributions that describe the behavior of the real system. For example, if the time to failure of a component, $t$, follows a Weibull distribution with scale parameter $\eta$ and shape parameter $\beta$ for which the cumulative distribution function (CDF), $F(t)$, is:

$$
F(t)=1-\exp \left[-\left(\frac{t}{\eta}\right)^{\beta}\right]
$$

then using a random number $x$ sampled from the $\mathrm{U}[0,1]$ distribution, the component failure time can be calculated using the expression:

$$
t=\eta[-\ln (x)]^{1 / \beta}
$$

Numerical algorithms may be used if a probability distribution does not have an analytical inverse function, for example the central limit theorem can be used to sample from a Normal distribution [27].

The information for calculating the performance measures is collected during the simulation of the computer model. For example, the number of mission ground aborts and air aborts are respectively equal to the number of tokens in the place 'Cancelled mission' and the number of times the transition 'Mission air abort' fires. With these data, the mission abort rate, a measure of fleet reliability and maintenance performance, can be calculated by [5]:

$$
\text { Mission abort rate }=\frac{\text { Air aborts }+ \text { Ground aborts }}{\text { Total missions required }} \times 100 \%
$$

Other fleet reliability and maintenance performance measures can be calculated in a similar way, by tracking the number of times and durations of certain places being marked during the performed simulations.

A number of simulations are performed and standard statistical techniques used to analyse the overall fleet performance. The confidence level of the simulation results depends on the number of simulations. Running a large number of simulations will lead to more accurate results, but could be time-consuming [28]. However, a large number of simulations may be unnecessary if the results reach a desired level of convergence, a threshold for which can be specified [28].

\section{Model Application}

To demonstrate the application of the proposed CPN framework, it is applied to an example fleet to investigate the effect of maintenance crews on fleet performance and to provide a guide for fleet managers to determine staffing level. The fleet consists of 50 identical and independent platforms, each of which is comprised of 5 binary-state LRUs that are connected in series. Each LRU consists of 2 different series-connected binary-state SRUs. There are 6 types of SRU in total. The fleet is assumed to perform fixed, identical, daily missions for 5 years. 45 missions must be performed by the fleet each day. Each mission is performed by one FMC platform, starting at 8am and lasting 16 hours. The maximum assignment time for each mission is 15 minutes. The fleet is assumed to employ a corrective maintenance policy and no preventive maintenance actions are performed. 
Table 3. Example fleet LRU parameters.

\begin{tabular}{|c|c|c|c|}
\hline LRU & $\begin{array}{c}\text { Child } \\
\text { SRUs }\end{array}$ & $\begin{array}{c}\text { Remove/Install } \\
\text { time (hour) }\end{array}$ & $\begin{array}{c}\text { I-level } \\
\text { repair } \\
\text { probability }\end{array}$ \\
\hline$L_{1}$ & $S_{1}, S_{3}$ & $\begin{array}{c}\text { Triangle } \\
(0.1,0.25,0.3)\end{array}$ & 0.8 \\
\hline$L_{2}$ & $S_{2}, S_{4}$ & $\begin{array}{c}\text { Uniform } \\
(0.5,0.8)\end{array}$ & 0.7 \\
\hline$L_{3}$ & $S_{3}, S_{5}$ & $\begin{array}{c}\text { Triangle } \\
(0.15,0.3,0.4)\end{array}$ & 0.9 \\
\hline$L_{4}$ & $S_{4}, S_{6}$ & $\begin{array}{c}\text { Triangle } \\
(0.4,0.5,0.6)\end{array}$ & 0.75 \\
\hline$L_{5}$ & $S_{5}, S_{6}$ & $\begin{array}{c}\text { Triangle } \\
(0.3,0.35,0.40)\end{array}$ & 0.85 \\
\hline
\end{tabular}

Table 4. Example fleet SRU parameters

\begin{tabular}{|c|c|c|c|c|}
\hline SRU & Failure time & $\begin{array}{c}\text { Remove/Install } \\
\text { time (hour) }\end{array}$ & $\begin{array}{c}\text { Repair time } \\
\text { (hour) }\end{array}$ & $\begin{array}{c}\text { I-level } \\
\text { repair } \\
\text { probability }\end{array}$ \\
\hline$S_{1}$ & $\begin{array}{c}\text { Weibull } \\
(\beta=1.3, \eta=2200)\end{array}$ & $\begin{array}{c}\text { Triangle } \\
(3,4,5)\end{array}$ & $\begin{array}{c}\text { Uniform } \\
(120,130)\end{array}$ & 0.5 \\
\hline$S_{2}$ & $\begin{array}{c}\text { Normal } \\
(\mu=1800, \sigma=23)\end{array}$ & $\begin{array}{c}\text { Uniform } \\
(5.5,6.5)\end{array}$ & $\begin{array}{c}\text { Uniform } \\
(75,80)\end{array}$ & 0.7 \\
\hline$S_{3}$ & $\begin{array}{c}\text { Lognormal, } \\
(\mu=7.6, \sigma=0.8)\end{array}$ & $\begin{array}{c}\text { Triangle } \\
(4,5,6)\end{array}$ & $\begin{array}{c}\text { Uniform } \\
(135,145)\end{array}$ & 0.4 \\
\hline$S_{4}$ & $\begin{array}{c}\text { Weibull } \\
(\beta=1.2, \eta=1950)\end{array}$ & $\begin{array}{c}\text { Triangle } \\
(3,4.5,5.5)\end{array}$ & $\begin{array}{c}\text { Triangle } \\
(95,100,105)\end{array}$ & 0.65 \\
\hline$S_{5}$ & $\begin{array}{c}\text { Weibull } \\
(\beta=1.5, \eta=2100)\end{array}$ & $\begin{array}{c}\text { Triangle } \\
(3.5,4.5,6)\end{array}$ & $\begin{array}{c}\text { Triangle } \\
(120,130,135)\end{array}$ & 0.55 \\
\hline$S_{6}$ & $\begin{array}{c}\text { Weibull } \\
(\beta=1.8, \eta=2000)\end{array}$ & $\begin{array}{c}\text { Triangle } \\
(3.8,4.6,5.5)\end{array}$ & $\begin{array}{c}\text { Triangle } \\
(110,120,128)\end{array}$ & 0.6 \\
\hline
\end{tabular}

Table 3 shows the distributions relating to LRU removal/installation time, the I-level repair probability of LRUs and the relationship between SRUs and LRUs. The distributions relating to SRU failure time, SRU removal/installation time and repair time, and the I-level repair probability of SRUs are shown in Table 4. The time to repair a failed SRU at the depot is assumed to be equal to that at the I-level maintenance organisation. The transportation time between the O-level and I-level maintenance organisations is a random variable which follows a Triangle distribution $(0.3,0.4,0.5)$ hours while that between I-level and D-level follows a Triangle distribution $(18,20,24)$ hours. The required number of maintenance crews to finish any maintenance action is one.

11 I-level staffing scenarios, where the numbers of I-level maintenance crews vary from 5 to 15 , are studied, assuming infinite O-level and D-level maintenance crews are employed and no spares are available. The duties of crews at I-level are the restoration of failed LRUs through SRU removal and installation, and SRU repair. When there are a limited number of Ilevel maintenance crews available, the maintenance actions at the I-level repair shop are performed in the following order:

1. All possible removals of failed SRUs,

2. All possible installations of spare SRUs,

3. All possible repairs of failed SRUs.

The fleet performance is measured by mission capable rate (MCR, proportion of time that platforms in the fleet are capable of performing missions in a given time interval) and mission abort rate (MAR, proportion of missions that are not performed successfully due to air aborts and ground aborts). 200 simulations were performed, although convergence of results for both performance indicators was observed by 100 simulations. Figures 56 and 57 summarise the average fleet performance under different staffing scenarios. The simulation results suggest that, for the modelled fleet, the fleet performance increases with the number of maintenance crews employed at the I-level maintenance organisation. This is expected since more failed LRUs and SRUs can be repaired in time when there are more I-level maintenance crews, which acts to increase the number of MC platforms and to decrease the number of ground 
aborts. In addition, when 11 crews are employed at I-level, employing more crews bring no benefits to fleet performance. Therefore, it may be not necessary to employ more than 11 maintenance crews at the I-level maintenance organisation when the budget is limited.

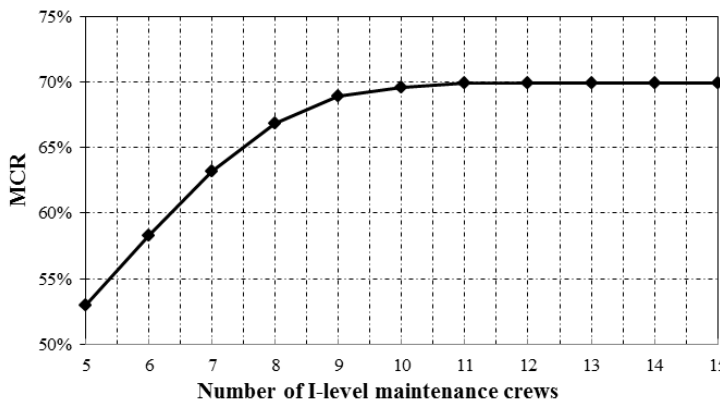

Figure 56. Change of MCR with number of I-level maintenance crews

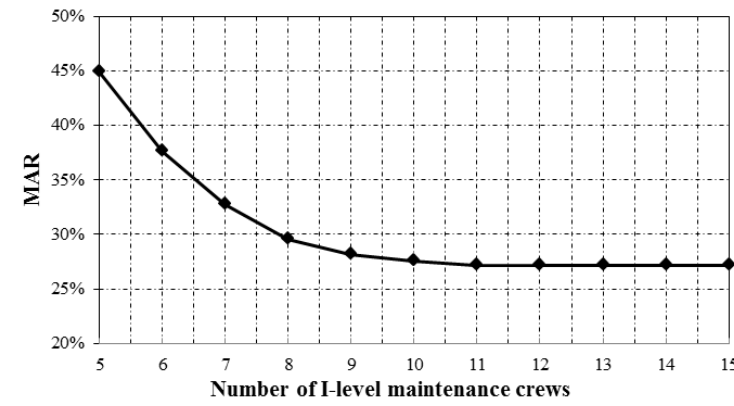

Figure 57. Change of MAR with number of I-level maintenance crews

\section{Conclusions}

- The CPN framework proposed in this paper allows the CPN method to be used to model fleet operation, aircraft failure, component failure and fleet maintenance processes.

- After inputting fleet parameters to the CPN model through setting the appropriate initial place markings, transition firing delays and arc expressions, the initialised CPN model provides an ideal framework for performing a Monte Carlo simulation of modelled fleets.

- Numerous fleet reliability and maintenance performance measures that are of interest to fleet managers can be obtained using information collected during the simulation process.

- The model can be efficiently modified and extended to model the effect of operation and maintenance factors on fleet performance due to its modular nature, which also facilitates its future extension to include further operation and maintenance factors that impact fleet performance.

- The CPN framework provides a powerful tool to assist fleet managers in designing, managing and optimising fleet maintenance systems.

\section{Reference}

[1] Deac V, Carstea G, Bagu C, Parvu F. The modern approach to industrial maintenance management. Inform Econ 2010;14(2):133-44.

[2] Sriram C, Haghani A. An optimisation model for aircraft maintenance scheduling and reassignment. Transport Res A-Pol 2003;37:29-48.

[3] Powell MJ. The effects of consolidating F-16 phase and cannibalisation aircraft on key maintenance indicators, M.S. thesis. United States Army Command and General Staff College; 1993.

[4] Friend CH. Aircraft maintenance management. Harlow: Longman; 1992.

[5] Dupuy MJ, Wesely DE, Jenkins CS. Airline fleet maintenance: trade-off analysis of alternate aircraft maintenance approaches. In: Proceedings of Systems and Information Engineering Design Symposium; 2011 Apr 29; Charlottesville, USA. New York: IEEE; 2011. p. 29-34.

[6] Varelis AG, Stamboulis YA, Adamides ED. A life-cycle system dynamic model of aircraft engine maintenance. In: Proceedings of the $20^{\text {th }}$ System Dynamic Conference; 2002 Jul 28-Aug 1; Palermo, Italy. New York: IEEE; 2002. p. 265-81.

[7] Mattila V, Virtanen K, Raivio T. Improving maintenance decision making in the Finnish air force through simulation. Interfaces 2008;38(3):187-201.

[8] Sheng J, Prescott DR. A hierarchical coloured petri net model of fleet maintenance with cannibalisation. Reliability Eng Syst Saf 2017;168:290-305.

[9] Safaei N, Bamjevic D, Jardine AKS. Workforce-constrained maintenance scheduling for aircraft fleet: a case study. Ann Oper Res 2010;186:295-316. 
[10] Yang J, Zhang F, Li K, Du J. Research on aeronautical component repair business optimization based on CTPN. In: Proceedings of 2012 International Conference on Computer Science and Electronics Engineering; 2012 Mar 23-25; Hangzhou, China. New York: IEEE; 2012. p. 660-4.

[11] Feng Q, Bi X , Zhao X, Chen Y, Sun B. Heuristic hybrid game approach for fleet condition-based maintenance planning. Reliability Eng Syst Saf 2017;157:166-76.

[12] Shell RE. A simulation study comparing multi-level maintenance concepts for the F16C/D dual mode transmitter, M.S. thesis. United States Air University; 1989.

[13] Wang Y. Simulation of aircraft two level maintenance concept based on petri net. In: Proceedings of International Conference on Quality, Reliability, Risk, Maintenance and Safety Engineering; 2012 Jun 15-18; Chengdu, China. New York: IEEE; 2012. p. 517-9.

[14] Tang X, Zhong S. Timed colored petri nets based modelling and scheduling of aeroengine maintenance. J Nat Sci 2006;4(2):46-51.

[15] Gopalan R. The aircraft maintenance base location problem. Eur J Oper Res 2014;236(2): 634-42.

[16] Alfedsson P. Optimisation of multi-echelon repairable item inventory systems with simultaneous location of repair facilities. Eur J Oper Res 1997; 99(3):584-95.

[17] Gallasch GE, Francis B, Moon C, Billington J. Modelling personnel within a defence logistics maintenance process. In: Proceedings of the $1^{\text {st }}$ International Conference on Simulation Tools and Techniques for Communcations, Networks and Systems; 2008 Mar 03-07; Marseille, France. Marseille: ISCT; 2008. p. 155-64.

[18] Sun Y, Chen X, Ren H, Jin Y, Liu Q. Ordering decision-making methods on spares parts for a new aircraft fleet based on a two-sample prediction. Reliability Eng Syst Saf 2016; 156:40-50.

[19] Qu C, Zhang L, Zhang B, Gao M. Maintenance resource scheduling modeling by petri net. In: Proceedings of $9^{\text {th }}$ International Conference on Electronic Measurement \& Instruments; 2009 Aug 16-19; Beijing, China. New York: IEEE; 2009. p. 4861-5.

[20] Wang S, Liu Y, Zhu D. Study on maintenance resource management model based on timed coloured petri net with inbihitor arcs. J Syst Eng Electron 2007;6: 170-3.

[21] Petri, CA. Kommunikation mit automaten (Communication with Machines), Ph.D. thesis. Darmstadt University of Technology; 1962.

[22] Chew S. Systems reliability modelling for phased missions with maintenance free operating periods, Ph.D. thesis. Loughborough University; 2010.

[23] Volovoi V. System-level maintenance policies via stochastic petri nets with aging tokens. In: Proceedings of Annual Reliability and Maintainability Symposium; 2007 Jan 22-25; Orlando, USA. New York: IEEE; 2007. p. 89-94.

[24] Jensen K, Kristensen LM. Coloured petri nets: modelling and validation of concurrent systems. Heidelberg: Springer; 2009.

[25] Gurler U, Kaya A. A maintenance policy for a system with multi-state components: an approximate solution. Reliability Eng Syst Saf 2002;76(2):117-27.

[26] Chew S, Dunnett SJ, Andrews JD. Phased mission modelling of systems with maintenance free operating periods using simulated petri nets. Reliability Eng Syst Saf 2008;93(7):980-94.

[27] Prescott DR. Safety modelling for the time limited dispatch of aircraft, Ph.D. thesis. Loughborough University; 2007.

[28] Le B. Modelling railway bridge asset management, Ph.D. thesis. University of Nottingham; 2013.

[29] Raychaudhuri S. Introduction to monte carlo simulation. In: Proceedings of Winter Simualtion Conference; 2008 Dec 7-10; Miami, USA. New York: IEEE; 2008. p. 91-100.

\section{Appendix: Acronyms}

$\begin{array}{llll}\text { CM } & \text { Corrective Maintenance } & \text { PM } & \text { Preventive Maintenance } \\ \text { LRU } & \text { Line Replaceable Unit } & \text { SRU } & \text { Shop Replaceable Unit } \\ \text { O-level } & \text { Organisation Level } & \text { I-level } & \text { Intermediate Level }\end{array}$


D-level Depot Level

DMT Dual Mode Transmitter

CPN Coloured Petri Nets

NMC Non Mission Capable

PMC Partially Mission Capable

FT
FiAF Finnish Air Force

PN Petri Nets

MC Mission Capable

FMC Fully Mission Capable

MEL Minimum Equipment List

TPM Time-based Preventive Maintenance 